
\title{
B Research S Sulure \\ Understand the role of exosomal miRNA in the development of breast cancer in patients with chronic HBV infection
}

Wenjun Xie ( $\square$ xiewenjun89@163.com )

Fujian Provincal Hospital

\section{Chun Chen}

Newcastle University

Qiang Lin

Fujian Provincal Hospital

Huashui Li

Fujian Provincal Hospital

\section{Research Article}

Keywords: Chronic HBV infection, exosomes, breast tumours, carcinoma, microRNA

Posted Date: April 21st, 2021

DOI: https://doi.org/10.21203/rs.3.rs-428432/v1

License: (c) (i) This work is licensed under a Creative Commons Attribution 4.0 International License. Read Full License 


\section{Abstract}

Background: Female chronic hepatitis B patients has shown a higher incidence of breast cancer with poorer prognosis, compared to the non-infected female patients. A significant difference in miRNAs expression in serum exosomes has been identified between female patients with chronic HBV infection and non-infected female. Whether this difference could contribute to the differential incidence and development of breast cancer in these female patients has not been fully understood.

Methods: Information of exosomal expression of miRNAs (expression profiles of GSE33857) were acquired from the Gene Expression Omnibus (GEO) database. Targeted genes of this miRNAs and the differentially expressed genes of breast cancer in GEPIA V2.0 database was used to obtain the differentially expressed genes (DEGs). Four analytic methods were performed, including functional annotation, protein-protein interaction (PPI) network and module construction, the clinical pathologic parameters analysis, alongsides prognosis analysis.

Results: In the serum exsomes, expression of miR-1237-3p and miR-363-3p were down-regulated whilst expression of miR-100-5p, miR-125b-5p, miR-1260a, miR-1287-5p, miR-148a-3p, miR-885-5p and miR-99a-5p were up-regulated in female chronic hepatitis B (CHB) patients. 228 up-regulated genes and 641 down-regulated genes were selected as DEGs. The DEGs involved in several biological processes ranging from cellular response to hormone stimulus. According to the main enriched Kyoto Encyclopedia of Genes and Genomes (KEGG) pathway was the pathways in cancer. Our analysis revealed that among the hub genes, CXCL11 was up-regulated and CXCL12, GNG2, LPAR1, GPR17, CXCL1, ADCY5, ADCY3, CX3CL1, S1PR1 were down-regulated. These genes expression levels were verified in UALCAN, Oncomine and HPA database. High expression level of CXCL11 and low expression levels of CXCL12, LPAR1, ADCY3 and CX3CL1 were positively correlated with some clinical pathological parameters that predicted worse prognosis. Kaplan-Meier (K-M) plotter analysis revealed that these genes that showed a low expression level were positively correlated with poor prognosis of breast cancer, and the high expression genes were negatively correlated with poor prognosis of breast cancer.

Conclusion: This bioinformatics analysis study explored the mechanisms of chronic HBV related occurrence and development of breast cancer by changing the expression of miRNAs of serum exosomes. It provided novel directions and therapeutic strategy for chronic HBV infection associated occurrence and development of breast cancer in female patients.

\section{Introduction}

Hepatitis B is a common infectious disease caused by the hepatitis B virus (HBV). It was estimated that there were 250 million population with chronic HBV infection worldwide in $2020^{[1]}$. Approximately one million people died from liver conditions, including liver cancer and cirrhosis that were associated to HBV infection ${ }^{[2]}$. In addition to liver cirrhosis and hepatocellular carcinoma, HBV infection could also contribute to the development of extrahepatic malignances, including lymphoma, cholangiocarcinoma and breast carcinoma ${ }^{[3]}$.

Breast cancer in a common malignant tumor in female, with an estimated number of 274,480 new cases and 42,170 death toll in $2020^{[4]}$. Studies have found a significant higher incidence ${ }^{[3]}$, an earlier age-of-onset ${ }^{[5]}$ and poorer prognosis ${ }^{[6]}$ of breast cancer in female HBV patients in comparison to those without HBV infection. However, mechanism of such association between breast cancer and chronic HBV infection has not been elucidated. Evidence suggested damages of hepatocytes that were caused by chronic inflammation from HBV-infection ${ }^{[7]}$ could lead to 
abnormal metabolism of sexual hormone, resulting in malignant changes of breast tissues ${ }^{[8]}$. Another study showed that HBV could cause directly induces malignancy via viral replication within breast cells ${ }^{[9]}$.

Exosomes are small vesicles with a size of 50-150nm in diameter derived from endosomes ${ }^{[10]}$. It is characterized with lipid bilayer and contains abundant RNA, DNA, proteins and lipids from cellular metabolism ${ }^{[11,12]}$. The lipid bilayer protects the exosomal contents from degradation and maintains the integrity of exosomes in serum ${ }^{[13]}$. Exosomes travel within the circulation system and could interact with tissues, affecting biological functions ${ }^{[14-16]}$. Roles of exosomes have been highlighted in several lines of cancer researches, including tumor growth, invasion and metastasis, immune escape, chemotherapy resistance and radiotherapy tolerance ${ }^{[17-19]}$. Moreover, the accessibility of exosome detection from serum samples allows it to become an ideal candidate for clinical biomarkers.

Recent study has identified differential expression level of exosomal miRNAs between HBV-infected and normal individual[ ${ }^{[20]}$. Moreover, alterations of miRNA levels in blood exosomes were found to be able to modify the progression of hepatocellular carcinoma ${ }^{[21]}$ and breast carcinoma ${ }^{[22]}$ respectively. However, weather or not HBV infection could promote breast carcinogensis via the alteration of exsomal miRNAs in patient's serum remains unknown. Here, we performed a comprehensive statistical analysis to identify the common exosomal miRNAs showing a differential expression level in HBV-infected individual and investigate if these miRNAs contribute to the expression changes and clinical outcomes in patients with breast cancer. Through search of potential genetic links between HBV infection and breast cancer, our study could provide novel insight of the regulation mechanism underlying the more rapid progression and poorer prognosis of breast cancer in HBV infected patient, for genetic targets development for diagnosis, evaluation of disease therapy and prognosis.

In this study, identification of exosomal miRNAs showing differential expression level in HBV infected patients was performed using GEO2R analysis ${ }^{[23]}$, with potential target genes screened by the mirWALK ${ }^{[24]}$. Intersection of these genes and breast cancer related genes indentified based on the GEPIA V2.0 data ${ }^{[25]}$ was analysed, generating the differentially expressed genes (DEGs). The DEGs was then used for G0 and KEGG analysis, constructing the PPI network analysis and the identification of hub genes for breast cancer. Expression of these hub genes were also verified using the Oncomine, UALCAN and HPA databases. Moreover, relationship between the identified hub genes with critical clinical pathological parameters and the prognosis of breast cancer were also investigated using the bcGenExMiner and the K-M plotter databases.

\section{Materials And Methods}

Chip data

GSE33857 was generated from Agilent-029297 Human miRNA Microarray v14 Rev.2 data. These data contained miRNAs data from 100 samples, including four patients with chronic HBV infection, 64 patients with chronic hepatitis C infection, 7 patients with non-alcoholic hepatitis, 12 healthy individuals and 13 huh7 liver cancer cells ${ }^{[23]}$.

Identification of DEGs

GEO2R is an interactive web tool provided by the National Center for Biotechnology Information (NCBI). This tool was used to analyze the differences in exsomal miRNAs between female with and without chronic HBV infection. In this study, a significantly different expression level of the miRNAs was defined based on a fold change $|\log F C|>1.5$ with $p$ < 0.01. MirWalk database (http://zmf.umm.uni-heidelberg.de/apps/zmf/mirwalk2/index.html) was used to analyze the predicted target genes for miRNAs. GEPIA (Gene Expression Profiling Interactive Analysis) V2.0 database

Page 3/34 
(http://gepia.cancerpku.cn/) was used to perform the screening to identify the significantly expressed genes in breast cancer tissues $^{[25]}(|\operatorname{logFC}|>1.0$ and $p<0.05)$. Venn diagram was used to present the intersection of up-regulated miRNAs target genes which showed a low expression level with the significantly low expressed genes in breast cancers, alongsides the down-regulated miRNAs target genes which showed a high expression level with the significantly high expressed genes in breast cancer.

Gene ontology and KEGG pathway analysis

Enrichr ${ }^{[26,27]}$ database (https://maayanlab.cloud/Enrichr/) was used to analyze the biological process (BP), cell component (CC), molecular function (MF) and KEGG pathway of the DEGs.

Identification of top-level modules and hub genes in the PPI networks

The Search Tool for the Retrieval of Interacting Genes (STRING, v11.0) (https ://strin g-db.org/) was used to analyze protein interactions between genes, and generate PPI network for visualization. Cytoscape software V3.7.1 (http://www.cytos cape.org) was used to screen the top-level modules in the PPI network based on the following criterial using the Molecular Complex Detection (MCODE): degree cut-off $=2$, node score cut-off $=0.2$, max depth $=$ 100 and k-score $=2$. A plug of the Cytoscape software, CytoHubba was installed in order to calculate the nodes of each protein, as well as the MCC algorithm to identify the top ten genes as hub genes.

Validation of relative mRNA expression levels of hub genes

To further validate the mRNA level of hub genes in breast cancer, we tested these differential expressions of genes from the UALCAN and Oncomine databases. UALCAN (http://ualcan.path.uab.edu/) contains the RNA sequence and the relative expression of genes related to normal and tumor tissues in 31 tumors ${ }^{[28]}$. Oncomine v4.5(https://www.oncomine.org/) which provides cancer microarray data sets and data mining functions for multiple cancer type was used to validate the implication of the potential gene target we identified in the breast tumor development and progression ${ }^{[29]}$.

Explore the expression level of hub genes in the human protein atlas database (HPA)

HPA V20.0(https://www.proteinatlas.org/) was an online free database that can provide abundant transcriptome and proteome data analysis for human normal or pathological tissues through RNA sequence analysis and immunohistochemistry ${ }^{[30]}$. This study explored the protein expression and distribution of hub genes in breast cancer tissues and normal tissues in HPA.

Explore the expression level of the hub genes to breast cancer clinicopathological parameters bc-GenExMiner $\mathrm{v} 4.15^{[31]}$ (http://bcgenex.ico.unicancer.fr) is a statistical mining tool which contains breast cancer transcriptomic data (DNA microarrays [ $n=10,716]$ and RNA-seq $[n=4,712]$. This database allows statistical analysis of gene expression, prognosis and correlation in breast cancer tissue. The data on this site was last updated in 06/2020. We analyzed the relationship between the hub genes and breast cancer clinicopathological parameters by using this database.

Survival analysis of hub genes

K-M plotter ${ }^{[32]}$ (https://kmplot.com/analysis/) was an online platform that can evaluate the effects of 54,000 genes (mRNA, miRNA, proteins) on the survival of 21 cancers, including breast cancer $(n=6234)$, ovarian cancer $(n=2190)$, lung cancer $(n=3452)$ and gastric cancer $(n=1440)$. The sources of the database include GEO, the Molecular Taxonomy of Breast Cancer International Consortium (METABRIC) and TCGA. The main purpose of this tool was to 
discover and verify survival-related biomarkers based on meta-analysis. The expression of genes was divided into high-expression group and low-expression group according to the median and $p<0.05$ indicates that the difference of prognosis was statistically significant.

\section{Results}

\section{Identification of exosomal miRNAs showing differential expression with HBV infection}

Using GSE33857 database, our analysis identified 251 miRNAs expressed differentially in serum samples from female chronic hepatitis $B(C H B)$ patients $(n=4)$, compared to controls $(n=12)($ Fig. $1 A)$. Among them, there were nine miRNAs showing significant changes in comparison to controls $(p<0.01)$, seven of which showed upregulation (fold change $\geq 1.5$ ), including miR-100-5p, miR-125b-5p, miR-1260a, miR-1287-5p, miR-148a-3p, miR-885-5p and miR99a-5p; two of which showed downregulation (fold change, $\leq-1.5$ ), including miR-1237-3p and hsa-miR-363-3p. Basing on the mirWALK datebase, we identified 3293 target genes that were associated to theses down-regulated miRNAs and 6260 genes associated to these up-regulated miRNAs (binding score >0.95) (Supplementary Table 1) .

\section{Integration with genes showing differential expression in breast cancer}

Using the GEPIA V2.0, 1416 up-regulated genes and 2134 down-regulated genes were indentified in breast samples from patients with breast cancer in comparison to controls (Supplementary Table 2). To find genetic integration between HBV-related exosome and breast cancer, intersection analysis was performed on the target genes identified showing differential expression in serum samples from patients with HBV-infection and breast cancer respectively. This analysis identified 228 common genes showing an increased expression level in the breast cancer that were associated to the HBV-related downregulated miRNAs (Fig. 1B). On the other hand, there were 640 common genes showing a decreased expression level that were associated to the upregulated miRNAs (Fig. 1C)(Supplementary Table 3).

\section{Pathway and network analysis of the common genes}

To understand the biological meanings of the identified common genes, GO enrichment and KEGG analysis was performed for pathway and network analysis. The common genes showing differential expression with HBV-infection and breast cancer were enriched for regulation of cellular response to hormone stimulus, signal transduction, epithelial cell proliferation, aorta morphogenesis, extracellular matrix organization, MAPK cascade, regulation of endothelial cell proliferation, and cytoskeleton organization, positive regulation of intracellular signal transduction, negative regulation of signal transduction in biological process (BP)(Fig. 2A, Table 1). The main cell component (CC) involved included integral components of plasma membrane, cytoskeleton, actin cytoskeleton, contractile actin filament bundle, stress fiber, actomyosin, platelet alpha granule membrane, focal adhesion, alongside axon and spindle midzone(Fig. 2B, Table 1).In addition, the main molecular function (MF) involved were actin binding, phosphatidylinositol-4,5-bisphosphate 3-kinase activity, actin filament binding, phosphatidylinositol bisphosphate kinase activity, transmembrane receptor protein kinase activity, phosphatidylinositol 3-kinase activity, protein kinase binding and kinase binding, protein kinase activity, and integrin binding (Fig. 2C, Table 1). At last, the main enriched KEGG pathways of these genes included pathways in cancer, ras signaling pathway, axon guidance, PI3K-Akt signaling pathway, proteoglycans in cancer, fluid shear stress and atherosclerosis, leukocyte transendothelial migration, Rap1 signaling pathway, focal adhesion and melanoma (Fig. 2D, Table 2). 
Page 6/34 
Table 1

The GO function enrichment analysis of the overlapping genes with differential expression in breast cancer

\begin{tabular}{|c|c|c|c|c|c|}
\hline GO & Category & Description & Count & $\%$ & P-value \\
\hline G0:0032870 & $\begin{array}{l}\text { GO Biological } \\
\text { Processes }\end{array}$ & cellular response to hormone stimulus & 17 & 0.21 & 4.63E-08 \\
\hline G0:0009966 & $\begin{array}{l}\text { GO Biological } \\
\text { Processes }\end{array}$ & regulation of signal transduction & 28 & 0.12 & $1.15 \mathrm{E}-06$ \\
\hline G0:0050678 & $\begin{array}{l}\text { GO Biological } \\
\text { Processes }\end{array}$ & regulation of epithelial cell proliferation & 14 & 0.19 & $2.20 \mathrm{E}-06$ \\
\hline G0:0035909 & $\begin{array}{l}\text { GO Biological } \\
\text { Processes }\end{array}$ & aorta morphogenesis & 7 & 0.44 & 2.31E-06 \\
\hline G0:0030198 & $\begin{array}{l}\text { GO Biological } \\
\text { Processes }\end{array}$ & extracellular matrix organization & 27 & 0.12 & $2.56 \mathrm{E}-06$ \\
\hline G0:0043408 & $\begin{array}{l}\text { GO Biological } \\
\text { Processes }\end{array}$ & regulation of MAPK cascade & 25 & 0.24 & $2.72 \mathrm{E}-06$ \\
\hline GO:1902533 & $\begin{array}{l}\text { GO Biological } \\
\text { Processes }\end{array}$ & $\begin{array}{l}\text { positive regulation of intracellular signal } \\
\text { transduction }\end{array}$ & 43 & 0.09 & 5.93E-06 \\
\hline GO:0009968 & $\begin{array}{l}\text { GO Biological } \\
\text { Processes }\end{array}$ & negative regulation of signal transduction & 30 & 0.11 & $6.48 \mathrm{E}-06$ \\
\hline G0:0001936 & $\begin{array}{l}\text { GO Biological } \\
\text { Processes }\end{array}$ & regulation of endothelial cell proliferation & 14 & 0.18 & $6.90 \mathrm{E}-06$ \\
\hline G0:0007010 & $\begin{array}{l}\text { GO Biological } \\
\text { Processes }\end{array}$ & cytoskeleton organization & 18 & 0.14 & 8.60E-06 \\
\hline G0:0005887 & $\begin{array}{l}\text { GO Cell } \\
\text { Component }\end{array}$ & integral component of plasma membrane & 111 & 0.08 & 4.59E-09 \\
\hline G0:0005856 & $\begin{array}{l}\text { GO Cell } \\
\text { Component }\end{array}$ & cytoskeleton & 48 & 0.09 & 7.78E-07 \\
\hline G0:0015629 & $\begin{array}{l}\text { GO Cell } \\
\text { Component }\end{array}$ & actin cytoskeleton & 31 & 0.11 & 5.10E-06 \\
\hline G0:0097517 & $\begin{array}{l}\text { GO Cell } \\
\text { Component }\end{array}$ & contractile actin filament bundle & 9 & 0.23 & 4.30E-05 \\
\hline G0:0001725 & $\begin{array}{l}\text { GO Cell } \\
\text { Component }\end{array}$ & stress fiber & 9 & 0.23 & 4.30E-05 \\
\hline G0:0042641 & $\begin{array}{l}\text { GO Cell } \\
\text { Component }\end{array}$ & actomyosin & 9 & 0.19 & 1.93E-04 \\
\hline G0:0031092 & $\begin{array}{l}\text { GO Cell } \\
\text { Component }\end{array}$ & platelet alpha granule membrane & 5 & 0.29 & $6.12 \mathrm{E}-04$ \\
\hline GO:0005925 & $\begin{array}{l}\text { GO Cell } \\
\text { Component }\end{array}$ & focal adhesion & 29 & 0.08 & $9.24 \mathrm{E}-04$ \\
\hline G0:0030424 & $\begin{array}{l}\text { GO Cell } \\
\text { Component }\end{array}$ & axon & 15 & 0.11 & 0.001232225 \\
\hline GO:0051233 & $\begin{array}{l}\text { GO Cell } \\
\text { Component }\end{array}$ & spindle midzone & 6 & 0.20 & 0.001603682 \\
\hline
\end{tabular}




\begin{tabular}{|c|c|c|c|c|c|}
\hline GO & Category & Description & Count & $\%$ & P-value \\
\hline G0:0003779 & $\begin{array}{l}\text { GO Molecular } \\
\text { Functions }\end{array}$ & actin binding & 32 & 0.13 & $6.66 \mathrm{E}-08$ \\
\hline G0:0046934 & $\begin{array}{l}\text { GO Molecular } \\
\text { Functions }\end{array}$ & $\begin{array}{l}\text { phosphatidylinositol-4,5-bisphosphate 3- } \\
\text { kinase activity }\end{array}$ & 13 & 0.19 & $6.14 \mathrm{E}-06$ \\
\hline GO:0051015 & $\begin{array}{l}\text { GO Molecular } \\
\text { Functions }\end{array}$ & actin filament binding & 18 & 0.14 & $9.62 \mathrm{E}-06$ \\
\hline GO:0052813 & $\begin{array}{l}\text { GO Molecular } \\
\text { Functions }\end{array}$ & $\begin{array}{l}\text { phosphatidylinositol bisphosphate kinase } \\
\text { activity }\end{array}$ & 13 & 0.18 & $1.01 \mathrm{E}-05$ \\
\hline GO:0019199 & $\begin{array}{l}\text { GO Molecular } \\
\text { Functions }\end{array}$ & $\begin{array}{l}\text { transmembrane receptor protein kinase } \\
\text { activity }\end{array}$ & 12 & 0.19 & $1.22 \mathrm{E}-05$ \\
\hline GO:0035004 & $\begin{array}{l}\text { GO Molecular } \\
\text { Functions }\end{array}$ & phosphatidylinositol 3-kinase activity & 13 & 0.17 & 2.17E-05 \\
\hline G0:0019901 & $\begin{array}{l}\text { GO Molecular } \\
\text { Functions }\end{array}$ & protein kinase binding & 42 & 0.08 & 2.93E-05 \\
\hline GO:0019900 & $\begin{array}{l}\text { GO Molecular } \\
\text { Functions }\end{array}$ & kinase binding & 36 & 0.09 & 7.90E-05 \\
\hline GO:0004672 & $\begin{array}{l}\text { GO Molecular } \\
\text { Functions }\end{array}$ & protein kinase activity & 41 & 0.08 & $1.36 \mathrm{E}-04$ \\
\hline GO:0005178 & $\begin{array}{l}\text { GO Molecular } \\
\text { Functions }\end{array}$ & integrin binding & 13 & 0.14 & 2.09E-04 \\
\hline
\end{tabular}

Table 2

The KEGG function enrichment analysis of the overlapping genes with differential expression in breast cancer

\begin{tabular}{|lllll|}
\hline Category & Description & Count & $\%$ & P-value \\
\hline KEGG_PATHWAY & Pathways in cancer & 47 & 0.090384615 & $3.07 \mathrm{E}-06$ \\
\hline KEGG_PATHWAY & Ras signaling pathway & 27 & 0.11637931 & $3.28 \mathrm{E}-06$ \\
\hline KEGG_PATHWAY & Axon guidance & 23 & 0.127071823 & $3.97 \mathrm{E}-06$ \\
\hline KEGG_PATHWAY & Pl3K-Akt signaling pathway & 35 & 0.098870056 & $5.48 \mathrm{E}-06$ \\
\hline KEGG_PATHWAY & Proteoglycans in cancer & 24 & 0.119402985 & $7.33 \mathrm{E}-06$ \\
\hline KEGG_PATHWAY & Fluid shear stress and atherosclerosis & 19 & 0.136690647 & $9.38 \mathrm{E}-06$ \\
\hline KEGG_PATHWAY & Leukocyte transendothelial migration & 16 & 0.142857143 & $2.69 \mathrm{E}-05$ \\
\hline KEGG_PATHWAY & Rap1 signaling pathway & 23 & 0.111650485 & $3.35 \mathrm{E}-05$ \\
\hline KEGG_PATHWAY & Focal adhesion & 22 & 0.110552764 & $5.72 \mathrm{E}-05$ \\
\hline KEGG_PATHWAY & Melanoma & 12 & 0.166666667 & $5.83 \mathrm{E}-05$ \\
\hline
\end{tabular}

To further explore the relationship between the differentially expressed genes (DEGs), we performed network analysis STRING database on the common genes showing differential expression with HBV-infection and breast cancer 
(Fig. 3A). Overall, the constructed PPI network was consisted of 868 nodes and 3285 edges. The top three modules within the network were identified via the MCODE analysis (Fig. 3B-D). Module 1 was composed of 16 nodes and 120 edges with the highest degree at 16.000. Module 2 was composed of 23 nodes and 102 edges with the highest degree at 9.273. Module 3 was composed of 35 nodes and 153 edges with the highest degree at 9.000.

\section{Identification and validation of hub genes}

The screening of hub genes related to the common genes showing differential expression with HBV-infection and breast cancer was performed using CytoHubba, and the top 10 genes which showed high correlation with the highest degree in the modules was identified (Fig. 3E). These hub genes included C-X-C Motif Chemokine Ligand 12(CXCL12), G-protein gamma 2 subunit(GNG2), lysophosphatidic acid receptor 1 (LPAR1), G protein-coupled receptor 17 (GPR17), C-X-C Motif Chemokine Ligand 1 (CXCL1), adenylate cyclase 5 (ADCY5), adenylate cyclase 3 (ADCY3), Fractalkine (FKN, CX3CL1), C-X-C Motif Chemokine Ligand 11 (CXCL11) and Sphingosine-1-Phosphate Receptor 1 (S1PR1).

Validation of these hub genes was firstly performed at a transcriptomic level using Oncomine and UALCAN. Overall, nine of the ten hub genes (CXCL12, GNG2, LPAR1, GPR17, CXCL1, ADCY5, ADCY3, CX3CL1, S1PR1) expressed decreasingly in the majority of cancer studies (Fold >1.5), with the exception of CXCL11 which showed remarkable increases in multiple type of cancer, including breast cancer in Oncomine databas(Fold > 1.5)(Fig. 4, Table 3). In breast cancer in particular, expression of eight hub genes (CXCL12, GNG2, LPAR1, GPR17, ADCY5, ADCY3, CX3CL1, S1PR1) showed an significantly decrease $(p<0.0001)$ whilst the expression of CXCL11 $(p<0.0001)$ was significantly increased in breast cancer tissue, according to the UALCAN database (Fig. 5) 
Table 3

Datasets of the hub genes in breast cancer (ONCOMINE database)

\begin{tabular}{|c|c|c|c|c|c|}
\hline Gene & Types of cancer vs normal & $\begin{array}{l}\text { Fole } \\
\text { change }\end{array}$ & $\mathrm{t}$-Test & P-value & Dataset \\
\hline \multirow[t]{15}{*}{ CXCL12 } & Invasive Ductal Breast Carcinoma & -5.734 & -41.69 & $\begin{array}{l}2.59 \mathrm{E}- \\
110\end{array}$ & Curtis et $\mathrm{al}^{30}$ \\
\hline & Medullary Breast Carcinoma & -6.063 & -17.075 & $\begin{array}{l}5.91 \mathrm{E}- \\
23\end{array}$ & \\
\hline & Invasive Lobular Breast Carcinoma & -3.738 & -17.01 & $\begin{array}{l}1.19 \mathrm{E}- \\
42\end{array}$ & \\
\hline & Invasive Ductal and Invasive Lobular Breast & -3.537 & -15.234 & $\begin{array}{l}2.63 \mathrm{E}- \\
13\end{array}$ & \\
\hline & Tubular Breast Carcinoma & -3.033 & -13.063 & $\begin{array}{l}1.71 \mathrm{E}- \\
23\end{array}$ & \\
\hline & Invasive Breast Carcinoma & -4.348 & -6.78 & $\begin{array}{l}4.97 \mathrm{E}- \\
07\end{array}$ & \\
\hline & Breast Carcinoma & -4.016 & -6.691 & $\begin{array}{l}4.08 \mathrm{E}- \\
06\end{array}$ & \\
\hline & Mucinous Breast Carcinoma & -5.087 & -10.593 & $\begin{array}{l}2.68 \mathrm{E}- \\
15\end{array}$ & \\
\hline & Invasive Ductal Breast Carcinoma & -6.730 & -25.411 & $\begin{array}{l}3.41 \mathrm{E}- \\
51\end{array}$ & TCGA Breast \\
\hline & Invasive Breast Carcinoma & -3.976 & -12.417 & $\begin{array}{l}6.37 \mathrm{E}- \\
24\end{array}$ & \\
\hline & Invasive Lobular Breast Carcinoma & -3.032 & -8.964 & $\begin{array}{l}6.88 \mathrm{E}- \\
13\end{array}$ & \\
\hline & Mixed Lobular and Ductal Breast Carcinoma & -3.846 & -7.112 & $\begin{array}{l}6.80 \mathrm{E}- \\
05\end{array}$ & \\
\hline & Lobular Breast Carcinoma & -2.791 & -6.306 & $\begin{array}{l}4.95 \mathrm{E}- \\
06\end{array}$ & Zhao et $\mathrm{a}^{31}$ \\
\hline & Invasive Ductal Breast Carcinoma & -5.095 & -12.634 & $\begin{array}{l}1.28 \mathrm{E}- \\
08\end{array}$ & \\
\hline & Invasive Breast Carcinoma Stroma & 18.556 & 16.597 & $\begin{array}{l}6.99 \mathrm{E}- \\
21\end{array}$ & Finak et al ${ }^{32}$ \\
\hline \multirow[t]{5}{*}{ GNG2 } & Mucinous Breast Carcinoma & -4.527 & -8.923 & $\begin{array}{l}5.41 \mathrm{E}- \\
06\end{array}$ & TCGA Breast \\
\hline & Mixed Lobular and Ductal Breast Carcinoma & -3.254 & -5.635 & $\begin{array}{l}6.90 \mathrm{E}- \\
05\end{array}$ & \\
\hline & Invasive Lobular Breast Carcinoma & -2.621 & -6.814 & $\begin{array}{l}4.37 \mathrm{E}- \\
10\end{array}$ & \\
\hline & Invasive Breast Carcinoma & -2.975 & -8.439 & $\begin{array}{l}1.12 \mathrm{E}- \\
13\end{array}$ & \\
\hline & Invasive Ductal Breast Carcinoma & -4.573 & -13.287 & $\begin{array}{l}4.15 \mathrm{E}- \\
21\end{array}$ & \\
\hline
\end{tabular}




\begin{tabular}{|c|c|c|c|c|c|}
\hline Gene & Types of cancer vs normal & $\begin{array}{l}\text { Fole } \\
\text { change }\end{array}$ & $\mathrm{t}$-Test & P-value & Dataset \\
\hline \multirow[t]{4}{*}{ LPAR1 } & Ductal Breast Carcinoma & -3.074 & -6.086 & $\begin{array}{l}8.33 \mathrm{E}- \\
07\end{array}$ & $\begin{array}{l}\text { Richardson et } \\
\mathrm{al}^{33}\end{array}$ \\
\hline & Invasive Ducatl Breast Carcinoma & -2.27 & -14.405 & $\begin{array}{l}5.98 \mathrm{E}- \\
29\end{array}$ & TCGA Breast \\
\hline & Medullary Breast Carcinoma & -2.317 & -7.909 & $\begin{array}{l}1.27 \mathrm{E}- \\
09\end{array}$ & Curtis et al ${ }^{30}$ \\
\hline & Mucinous Breast Carcinoma & -2.101 & -9.037 & $\begin{array}{l}7.90 \mathrm{E}- \\
13\end{array}$ & \\
\hline \multirow[t]{8}{*}{ CXCL1 } & Lobular Breast Carcinoma & -5.281 & -11.323 & $\begin{array}{l}5.33 \mathrm{E}- \\
06\end{array}$ & Sorlie et al ${ }^{34}$ \\
\hline & Ductal Breast Carcinoma & -6.82 & -12.515 & $\begin{array}{l}5.27 \mathrm{E}- \\
08\end{array}$ & \\
\hline & Lobular Breast Carcinoma & -4.4 & -9.896 & $\begin{array}{l}9.00 \mathrm{E}- \\
05\end{array}$ & Sorlie et al ${ }^{35}$ \\
\hline & Ductal Breast Carcinoma & -7.644 & -11.854 & $\begin{array}{l}6.77 \mathrm{E}- \\
09\end{array}$ & \\
\hline & Invasive Ducatl Breast Carcinoma & -8.437 & -5.111 & $\begin{array}{l}4.66 \mathrm{E}- \\
05\end{array}$ & Ma et al ${ }^{36}$ \\
\hline & Ductal Breast Carcinoma & -6.64 & -8.949 & $\begin{array}{l}5.93 \mathrm{E}- \\
05\end{array}$ & Perou et $\mathrm{al}^{37}$ \\
\hline & Invasive Lobular Breast Carcinoma & -5.38 & -8.731 & $\begin{array}{l}3.62 \mathrm{E}- \\
13\end{array}$ & TCGA Breast \\
\hline & Invasive Ductal Breast Carcinoma & -6.05 & -14.245 & $\begin{array}{l}9.30 \mathrm{E}- \\
30\end{array}$ & \\
\hline ADCY5 & Invasive Ductal Breast Carcinoma & -4.106 & -5.419 & $\begin{array}{l}4.13 \mathrm{E}- \\
06\end{array}$ & $\begin{array}{l}\text { Radvanyi et } \\
\mathrm{al}^{38}\end{array}$ \\
\hline \multirow[t]{6}{*}{ ADCY3 } & Invasive Ductal Breast Carcinoma & -2.306 & -38.362 & $\begin{array}{l}9.35 \mathrm{E}- \\
100\end{array}$ & Curtis et al ${ }^{30}$ \\
\hline & Invasive Lobular Breast Carcinoma & -2.19 & -21.264 & $\begin{array}{l}6.51 \mathrm{E}- \\
58\end{array}$ & \\
\hline & Invasive Ductal and Invasive Lobular Breast & -2.356 & -20.889 & $\begin{array}{l}2.46 \mathrm{E}- \\
45\end{array}$ & \\
\hline & Tubular Breast Carcinoma & -2.406 & -17.919 & $\begin{array}{l}5.08 \mathrm{E}- \\
32\end{array}$ & \\
\hline & Invasive Breast Carcinoma & -2.189 & -8.887 & $\begin{array}{l}4.73 \mathrm{E}- \\
09\end{array}$ & \\
\hline & Breast Carcinoma & -2.269 & -6.719 & $\begin{array}{l}5.48 \mathrm{E}- \\
06\end{array}$ & \\
\hline CX3CL1 & Mixed Lobular and Ductal Breast Carcinoma & -2.008 & -8.438 & $\begin{array}{l}2.23 \mathrm{E}- \\
12\end{array}$ & TCGA Breast \\
\hline
\end{tabular}




\begin{tabular}{|c|c|c|c|c|c|}
\hline Gene & Types of cancer vs normal & $\begin{array}{l}\text { Fole } \\
\text { change }\end{array}$ & $\mathrm{t}$-Test & P-value & Dataset \\
\hline & Male Breast Carcinoa & -3.108 & -17.039 & $\begin{array}{l}7.63 \mathrm{E}- \\
17\end{array}$ & \\
\hline & Invasive Ducatl Breast Carcinoma & -6.639 & -21.53 & $\begin{array}{l}6.08 \mathrm{E}- \\
43\end{array}$ & \\
\hline & Invasive Breast Carcinoma & -4.574 & -12.712 & $\begin{array}{l}5.12 \mathrm{E}- \\
25\end{array}$ & \\
\hline & Invasive Lobular Breast Carcinoma & -2.350 & -9.43 & $\begin{array}{l}6.60 \mathrm{E}- \\
15\end{array}$ & \\
\hline & Invasive Ducatl Breast Carcinoma & -3.714 & -4.751 & $\begin{array}{l}7.37 \mathrm{E}- \\
05\end{array}$ & $\begin{array}{l}\text { Radvanyi et } \\
\mathrm{al}^{38}\end{array}$ \\
\hline & Ductal Breast Carcinoma & -9.607 & -9.446 & $\begin{array}{l}1.41 \mathrm{E}- \\
05\end{array}$ & Perou et $\mathrm{al}^{37}$ \\
\hline & Ductal Breast Carcinoma & -9.119 & -10.194 & $\begin{array}{l}4.25 \mathrm{E}- \\
10\end{array}$ & $\begin{array}{l}\text { Richardson et } \\
\text { al }^{33}\end{array}$ \\
\hline & Invasive Ducatl Breast Carcinoma Epithelia & -25.610 & -6.262 & $\begin{array}{l}4.57 \mathrm{E}- \\
05\end{array}$ & Ma et al ${ }^{36}$ \\
\hline & Tubular Breast Carcinoma & -5.442 & -16.773 & $\begin{array}{l}2.06 \mathrm{E}- \\
36\end{array}$ & Curtis et $\mathrm{al}^{30}$ \\
\hline & Mucinous Breast Carcinoma & -5.875 & -14.955 & $\begin{array}{l}1.28 \mathrm{E}- \\
25\end{array}$ & \\
\hline & Invasive Lobular Breast Carcinoma & -4.279 & -16.479 & $\begin{array}{l}8.34 \mathrm{E}- \\
44\end{array}$ & \\
\hline & $\begin{array}{l}\text { Invasive Ductal and Invasive Lobular Breast } \\
\text { Carcinoma }\end{array}$ & -5.319 & -15.410 & $\begin{array}{l}1.15 \mathrm{E}- \\
34\end{array}$ & \\
\hline & Invasive Ducatl Breast Carcinoma & -4.960 & -24.263 & $\begin{array}{l}7.62 \mathrm{E}- \\
58\end{array}$ & \\
\hline & Breast Carcinoma & -4.460 & -6.528 & $\begin{array}{l}4.48 \mathrm{E}- \\
06\end{array}$ & \\
\hline & Medullary Breast Carcinoma & -2.845 & -7.190 & $\begin{array}{l}2.26 \mathrm{E}- \\
09\end{array}$ & \\
\hline CXCL11 & Invasive Ducatl Breast Carcinoma & 10.712 & 5.279 & $\begin{array}{l}2.81 \mathrm{E}- \\
05\end{array}$ & $\begin{array}{l}\text { Karnoub et } \\
\mathrm{al}^{39}\end{array}$ \\
\hline & Ductal Breast Carcinoma & 12.741 & 7.494 & $\begin{array}{l}1.52 \mathrm{E}- \\
09\end{array}$ & $\begin{array}{l}\text { Richardson et } \\
\mathrm{al}^{33}\end{array}$ \\
\hline & Invasive Breast Carcinoma & 8.811 & 13.055 & $\begin{array}{l}1.69 \mathrm{E}- \\
25\end{array}$ & TCGA Breast \\
\hline & Invasive Ducatl Breast Carcinoma & 5.867 & 15.594 & $\begin{array}{l}2.08 \mathrm{E}- \\
31\end{array}$ & \\
\hline & Invasive Lobular Breast Carcinoma & 5.268 & 7.777 & $\begin{array}{l}8.07 \mathrm{E}- \\
11\end{array}$ & \\
\hline
\end{tabular}




\begin{tabular}{|c|c|c|c|c|c|}
\hline Gene & Types of cancer vs normal & $\begin{array}{l}\text { Fole } \\
\text { change }\end{array}$ & $\mathrm{t}$-Test & P-value & Dataset \\
\hline \multirow[t]{3}{*}{ S1PR1 } & Invasive Ductal Breast Carcinoma Stroma & -2.958 & -6.441 & $\begin{array}{l}2.35 \mathrm{E}- \\
06\end{array}$ & Ma et $a^{36}$ \\
\hline & Tubular Breast Carcinoma & -2.051 & -14.313 & $\begin{array}{l}1.67 \mathrm{E}- \\
32\end{array}$ & Curtis et $\mathrm{al}^{30}$ \\
\hline & Invasive Breast Carcinoma & -2.030 & -7.920 & $\begin{array}{l}4.64 \mathrm{E}- \\
09\end{array}$ & \\
\hline & Medullary Breast Carcinoma & -2.138 & -9.752 & $\begin{array}{l}1.39 \mathrm{E}- \\
13\end{array}$ & \\
\hline & Invasive Ductal Breast Carcinoma & -2.153 & -19.997 & $\begin{array}{l}2.33 \mathrm{E}- \\
45\end{array}$ & \\
\hline & Invasive Ductal Breast Carcinoma & -4.289 & -15.878 & $\begin{array}{l}3.35 \mathrm{E}- \\
26\end{array}$ & TCGA Breast \\
\hline & Invasive Breast Carcinoma & -2.743 & -8.538 & $\begin{array}{l}1.50 \mathrm{E}- \\
14\end{array}$ & \\
\hline & Invasive Lobular Breast Carcinoma & -2.437 & -6.583 & $\begin{array}{l}2.41 \mathrm{E}- \\
09\end{array}$ & \\
\hline
\end{tabular}

Next, the immunohistochemical images of breast cancer acquired from the HPA database demonstrated the differential protein expression level of the ten hub genes between breast cancer tissue and normal breast tissue (Fig. 6). These showed that CXCL12 was low expressed in breast cancer tissue and normal breast tissue. GNG2 was low expressed in breast cancer tissue and medium expressed in normal breast tissue. GPR17 was not detected in breast cancer tissues and normal breast tissue. ADCY 5 was low expressed in breast cancer tissue and medium expressed in normal breast tissue. ADCY 3 was low expressed in breast cancer tissue and normal breast tissue. CX3CL1 was medium expressed in breast cancer tissues and highly expressed in normal breast tissue. CXCL11 was medium expressed in breast cancer tissues and low expressed in normal breast tissue. S1PR1 was low expression in breast cancer tissue and medium expressed in normal breast tissue. We did not manage to find images for LPAR1 and CXCL1 from the HPA dataset.

\section{The relationship between the hub genes and the key clinical parameters of breast cancer}

To provide clinical insights, we investigated the relationship between the hub genes identified with significant differential expression with HBV-infection and breast cancer, and critical clinical indicators that are routinely applied to estimate the prognosis of breast cancer, by using univariate Cox proportional hazards model in bc-GenExMiner database. These indicators included age, the estrogen receptor (ER), the progesterone receptor (PR) and the human epidermal growth factor receptor 2 (HER-2), alongside the lymph node metastasis.

In aged breast cancer patients ( $>51$ years old), $A D C Y 5(n=2285, p<0.0001)$, a significant higher mRNA expression levels whilst CXCL11 $(n=2812, p=0.001), \operatorname{CXCL} 12(n=2844, p<0.001), \operatorname{GNG} 2(n=2215, p<0.01), \operatorname{CXCL} 1(n=2844$, $p<0.001), \operatorname{ADCY} 3(n=2783, p<0.0001), C X 3 C L 1(n=2844, p<0.0001)$ showed a significant lower mRNA levels (Table 4). ER positive status was significantly positively correlated with CXCL12 $(n=6597, p<0.0001), \operatorname{LPAR} 1(n=$ $6597, p<0.0001), \operatorname{ADCY} 5(n=4652, p<0.0001)$ expression levels and negatively correlated with $\operatorname{CXCL11}(n=6310, p<$ 
0.0001), GNG2( $n=4872, p<0.001), \operatorname{CXCL1}(n=6597, p<0.0001), \operatorname{ADCY} 3(n=6506, p<0.0001), \operatorname{CX} 3 \operatorname{CL} 1(n=6597, p<$ $0.0001)$ expression levels. PR positive status was positively correlated with $\operatorname{CXCL} 12(n=3224, p<0.0001)$, LPAR1 ( $n=$ 3224, $p<0.0001)$ expression levels and negatively correlated with CXCL11 $(n=3034, p<0.0001), G N G 2(n=2761, p<$ 0.0002), GPR17( $n=3034, p=0.02) \operatorname{CXCL1}(n=3224, p<0.0001), \operatorname{ADCY} 5(n=2461, p<0.0001), \operatorname{ADCY} 3(n=3224, p<$ 0.0001), CX3CL1 $(n=3224, p<0.0001)$, S1PR1 $(n=3224, p<0.01)$. Similarly, HER-2 positive status was positively correlated with CXCL11 $(n=683, p<0.0001), \operatorname{CXCL1}(n=683, p<0.0001), \operatorname{ADCY} 5(n=642, p=0.019), \operatorname{CX3CL} 1(n=683$, $p<0.0001)$ expression levels and negatively correlated with CXCL12 $(n=683, p=0.02), \operatorname{LPAR} 1(n=683, p<0.0001)$, ADCY3 $(n=643, p<0.0001)$. Moreover, Triple-negative (ER+, PR+, HER-2 negative) status was positively correlated with CXCL11 ( $n=848, p<0.0001), G N G 2(n=671, p=0.0043), \operatorname{GPR} 17(n=848, p=0.030), \operatorname{CXCL1}(n=848, p<0.0001)$, $\operatorname{ADCY} 3(n=848, p<0.0001), C X 3 C L 1(n=848, p<0.0001)$ expression levels and negatively correlated with $\operatorname{CXCL} 12(n=$ 848, $p<0.0001), \operatorname{LPAR} 1(n=848, p<0.0001), \operatorname{ADCY5}(n=671, p<0.0001)$ expression levels (Table 4). Lymph node metastasis rate showed significant positive correlations with CXCL11 $(n=3458, p<0.001)$, LPAR1 $(n=3429, p<0.01)$ expression levels and negatively correlated with ADCY3 $(n=3429, p<0.001), \operatorname{CX} 3 C L 1(n=3511, p<0.01)$ expression levels (Table 4). 
Table 4

The relationship between the hub genes and the clinicopathologic parameters of breast cancer (bc-GenExMiner v4.1)

\begin{tabular}{|c|c|c|c|c|c|c|c|c|c|c|}
\hline \multirow[t]{2}{*}{ Parameters } & \multicolumn{2}{|l|}{ CXCL12 } & \multicolumn{2}{|l|}{ GNG2 } & \multicolumn{2}{|l|}{ LPAR1 } & \multicolumn{2}{|l|}{ GPR17 } & \multicolumn{2}{|l|}{ CXCL1 } \\
\hline & mRNA & $\begin{array}{l}\mathrm{P}- \\
\text { value }\end{array}$ & mRNA & $\begin{array}{l}\mathrm{P}- \\
\text { value }\end{array}$ & mRNA & $\begin{array}{l}\mathrm{P}- \\
\text { value }\end{array}$ & mRNA & P-value & mRNA & $\begin{array}{l}\mathrm{P}- \\
\text { value }\end{array}$ \\
\hline \multicolumn{11}{|l|}{ Age } \\
\hline$\leq 51$ & ref & $\begin{array}{l}p= \\
0.0002\end{array}$ & ref & $\begin{array}{l}p= \\
0.0092\end{array}$ & - & $\begin{array}{l}p= \\
0.6050\end{array}$ & - & $\begin{array}{l}p= \\
0.6108\end{array}$ & ref & $\begin{array}{l}p< \\
0.0001\end{array}$ \\
\hline$>51$ & $\downarrow$ & & $\downarrow$ & & - & & - & & $\downarrow$ & \\
\hline \multicolumn{11}{|l|}{$\begin{array}{l}\text { Nodal } \\
\text { status }\end{array}$} \\
\hline negative & - & $\begin{array}{l}p= \\
0.1709\end{array}$ & - & $\begin{array}{l}p= \\
0.1230\end{array}$ & ref & $\begin{array}{l}p= \\
0.0016\end{array}$ & - & & - & \\
\hline positive & - & & - & & $\uparrow$ & & - & $\begin{array}{l}p^{-} \\
=0.9069\end{array}$ & - & $\begin{array}{l}p= \\
0.0664\end{array}$ \\
\hline \multicolumn{11}{|l|}{$\mathrm{ER}(\mathrm{IHC})$} \\
\hline negative & ref & $\begin{array}{l}p< \\
0.0001\end{array}$ & ref & $\begin{array}{l}p= \\
0.0003\end{array}$ & ref & & - & & ref & $\begin{array}{l}p< \\
0.0001\end{array}$ \\
\hline positive & $\uparrow$ & & $\downarrow$ & & $\uparrow$ & $\begin{array}{l}p< \\
0.0001\end{array}$ & - & $\begin{array}{l}p= \\
0.0558\end{array}$ & $\downarrow$ & \\
\hline \multicolumn{11}{|l|}{$\mathrm{PR}(\mathrm{IHC})$} \\
\hline negative & & $\begin{array}{l}p< \\
0.0001\end{array}$ & ref & & ref & $\begin{array}{l}p< \\
0.0001\end{array}$ & ref & & ref & \\
\hline positive & $\uparrow$ & & $\downarrow$ & $\begin{array}{l}p< \\
0.0001\end{array}$ & $\uparrow$ & & $\downarrow$ & $\begin{array}{l}p= \\
0.0200\end{array}$ & $\downarrow$ & $\begin{array}{l}p< \\
0.0001\end{array}$ \\
\hline \multicolumn{11}{|l|}{ HER2(IHC) } \\
\hline negative & ref & $\begin{array}{l}p= \\
0.0202\end{array}$ & - & & ref & $\begin{array}{l}p< \\
0.0001\end{array}$ & - & & ref & $\begin{array}{l}p< \\
0.0001\end{array}$ \\
\hline positive & $\downarrow$ & & - & $\begin{array}{l}p= \\
0.1968\end{array}$ & $\downarrow$ & & - & $\begin{array}{l}p= \\
0.5521\end{array}$ & $\uparrow$ & \\
\hline \multicolumn{11}{|c|}{$\begin{array}{l}\text { Triple-negative } \\
\text { status }\end{array}$} \\
\hline Not & ref & $\begin{array}{l}p< \\
0.0001\end{array}$ & ref & $\begin{array}{l}p= \\
0.0043\end{array}$ & ref & $\begin{array}{l}p< \\
0.0001\end{array}$ & ref & $\begin{array}{l}p= \\
0.0299\end{array}$ & ref & $\begin{array}{l}\mathrm{p}< \\
0.0001\end{array}$ \\
\hline TNBC & $\downarrow$ & & $\uparrow$ & & $\downarrow$ & & $\uparrow$ & & $\uparrow$ & \\
\hline \multirow[t]{2}{*}{ Parameters } & ADCY5 & & ADCY3 & & CX3CL1 & & CXCL11 & & S1PR1 & \\
\hline & mRNA & $\begin{array}{l}\text { P- } \\
\text { value }\end{array}$ & mRNA & $\begin{array}{l}\mathrm{P}- \\
\text { value }\end{array}$ & mRNA & $\begin{array}{l}\text { P- } \\
\text { value }\end{array}$ & mRNA & P-value & mRNA & $\begin{array}{l}\text { P- } \\
\text { value }\end{array}$ \\
\hline \multicolumn{11}{|l|}{ Age } \\
\hline$\leq 51$ & ref & $\begin{array}{l}\mathrm{p}< \\
0.0001\end{array}$ & ref & $\begin{array}{l}\mathrm{p}< \\
0.0001\end{array}$ & ref & $\begin{array}{l}\mathrm{p}< \\
0.0001\end{array}$ & ref & $\begin{array}{l}p= \\
0.0010\end{array}$ & - & $\begin{array}{l}p= \\
0.9688\end{array}$ \\
\hline
\end{tabular}




\begin{tabular}{|c|c|c|c|c|c|c|c|c|c|c|}
\hline \multirow[t]{2}{*}{ Parameters } & \multicolumn{2}{|c|}{ CXCL12 } & \multicolumn{2}{|l|}{ GNG2 } & \multicolumn{2}{|l|}{ LPAR1 } & \multicolumn{2}{|l|}{ GPR17 } & \multicolumn{2}{|l|}{ CXCL1 } \\
\hline & mRNA & $\begin{array}{l}\text { P- } \\
\text { value }\end{array}$ & mRNA & $\begin{array}{l}\mathrm{P}- \\
\text { value }\end{array}$ & mRNA & $\begin{array}{l}\mathrm{P}- \\
\text { value }\end{array}$ & mRNA & P-value & mRNA & $\begin{array}{l}\mathrm{P} \text { - } \\
\text { value }\end{array}$ \\
\hline$>51$ & $\uparrow$ & & $\downarrow$ & & $\downarrow$ & & $\downarrow$ & & - & \\
\hline \multicolumn{11}{|l|}{$\begin{array}{l}\text { Nodal } \\
\text { status }\end{array}$} \\
\hline negative & - & & ref & $\begin{array}{l}\mathrm{p}= \\
0.0009\end{array}$ & ref & $\begin{array}{l}\mathrm{p}= \\
0.0052\end{array}$ & ref & $\begin{array}{l}p= \\
0.0002\end{array}$ & - & $\begin{array}{l}p= \\
0.6140\end{array}$ \\
\hline positive & - & $\begin{array}{l}p= \\
0.1407\end{array}$ & $\downarrow$ & & $\downarrow$ & & $\uparrow$ & & - & \\
\hline \multicolumn{11}{|l|}{$\mathrm{ER}(\mathrm{IHC})$} \\
\hline negative & ref & $\begin{array}{l}\mathrm{p}< \\
0.0001\end{array}$ & ref & $\begin{array}{l}\mathrm{p}< \\
0.0001\end{array}$ & ref & $\begin{array}{l}\mathrm{p}< \\
0.0001\end{array}$ & ref & $\begin{array}{l}\mathrm{p}< \\
0.0001\end{array}$ & - & $\begin{array}{l}p= \\
0.3491\end{array}$ \\
\hline positive & $\uparrow$ & & $\downarrow$ & & $\downarrow$ & & $\downarrow$ & & - & \\
\hline \multicolumn{11}{|l|}{$\mathrm{PR}(\mathrm{IHC})$} \\
\hline negative & ref & & ref & $\begin{array}{l}\mathrm{p}< \\
0.0001\end{array}$ & ref & $\begin{array}{l}\mathrm{p}< \\
0.0001\end{array}$ & ref & $\begin{array}{l}\mathrm{p}< \\
0.0001\end{array}$ & ref & $\begin{array}{l}p= \\
0.0011\end{array}$ \\
\hline positive & $\downarrow$ & $\begin{array}{l}p= \\
0.0480\end{array}$ & $\downarrow$ & & $\downarrow$ & & $\downarrow$ & & $\downarrow$ & \\
\hline \multicolumn{11}{|l|}{ HER2(IHC) } \\
\hline negative & ref & & ref & $\begin{array}{l}\mathrm{p}< \\
0.0001\end{array}$ & ref & $\begin{array}{l}\mathrm{p}< \\
0.0001\end{array}$ & ref & $\begin{array}{l}\mathrm{p}< \\
0.0001\end{array}$ & - & $\begin{array}{l}p= \\
0.1189\end{array}$ \\
\hline positive & $\uparrow$ & $\begin{array}{l}p= \\
0.0193\end{array}$ & $\downarrow$ & & $\uparrow$ & & $\uparrow$ & & - & \\
\hline \multicolumn{11}{|c|}{$\begin{array}{l}\text { Triple-negative } \\
\text { status }\end{array}$} \\
\hline Not & ref & $\begin{array}{l}\mathrm{p}< \\
0.0001\end{array}$ & ref & $\begin{array}{l}\mathrm{p}< \\
0.0001\end{array}$ & ref & $\begin{array}{l}\mathrm{p}< \\
0.0001\end{array}$ & ref & $\begin{array}{l}\mathrm{p}< \\
0.0001\end{array}$ & - & $\begin{array}{l}p= \\
0.1986\end{array}$ \\
\hline TNBC & $\downarrow$ & & $\uparrow$ & & $\uparrow$ & & $\uparrow$ & & - & \\
\hline
\end{tabular}

\section{Survival analysis of hub genes expression in patients with breast cancer}

In addition, relationship between the ten hub genes and the survival rate of breast cancer has been analysed to aid the understanding of the prognostic value of the ten hub genes. The survival analysis was performed using K-M plotter. The relatively low expression of GNG2 $(n=943, p<0.0001)$, LPAR1 $(n=1879, p<0.01), \operatorname{ADCY} 3(n=1879, p<$ $0.01)$ and high expression of $\operatorname{CXCL} 12(n=1879, p<0.001)$ was significantly associated with the poor overall survival (OS) (Fig. 7). The relatively low expression expression of GNG2 $(n=2032, p<0.001)$, LPAR1 $(n=4929, p<0.001)$, GPR17 ( $n=4929, p<0.0001)$, CXCL1 ( $=4929, p<0.001)$, ADCY5 $(n=2032, p<0.0001), \operatorname{ADCY3}(n=4929, p<0.0001)$, 
CXCL11 $(n=4929, p<0.001)$, S1PR1 $(n=4929, p<0.0001)$ and high expression of CXCL12 $(n=4929, p<0.0001)$ was associated with the poor recurrence free survival (RFS) (Fig. 8).

\section{Discussion}

Breast cancer was a common malignant tumor in female population, with an increasing prevalence in the past decade[33]. It becomes a major heath threaten to females and the public health worldwide, though clinical therapies including surgery, radiotherapy, chemotherapy and targeted therapy for breast cancer have been rapidly developing. Several epidemiological studies had highlighted an increased incidence[3] and bad prognosis[34] of breast cancer in female patients with chronic HBV infection. Therefore, this study investigated the mechanism that lies behind such association by analysing the involvement of HBV-related exosomal miRNA in the changes of gene expression with breast cancer. Via the identification of the key genetic targets that mediated the effects of HBV infection on the progression breast carcinoma, our results provided novel targets for the development of therapeutic strategy and prognostic biomarkers for breast cancer.

Firstly, our results found that miRNAs from the serum exosomes of female CHB patients, expression of hsa-miR-363 and hsa-miR-1237 showed significant down-regulation hsa-miR-1260, hsa-miR-100, hsa-miR-1287, hsa-miR-148a, hsa-miR-99a, hsa-miR-125b, hsa-miR-885-5p was significant up-regulation, in compared to uninfected individuals. Exosomes played critical roles in signal transmission within the human body through endocytosis and absorbance of organs, and has been implicated in occurrence and development of carcinoma ${ }^{[19]}$. Our hypothesis was that the changes of exosomal miRNAs that were indued by the chronic HBV infection affects breast cells via the endocytososis of exosomes in the circulatory system. This could lead to changes of related gene expression in the breast cancer cells, triggering the process of cainogenesis.

To prove this hypothesis, we identified the potential target genes of those miRNAs that showed a differential expression in CHB patients, which were then cross-analysed with genes showing differentially expression in breast cancer.

Through GO analysis, we found that CC was mainly located in the cytoplasmic membrane components, cytoskeleton and actin skeleton. These components were associated with tumor cell invasion and metastasis, cell adhesion ${ }^{[35,36]}$ and tumor cell immune tolerance ${ }^{[37]}$. BP were mainly concentrated in cellular response to hormone stimulus, regulation of signal transduction ${ }^{[38]}$ and regulation of epithelial cell proliferation ${ }^{[39]}$. These biological process were closely related to the occurrence and development of breast cancer. MF cluster analysis showed that the DEGs functions mainly include a variety of actins ${ }^{[40]}$, integrins ${ }^{[41]}$, and kinase anchoring and activity ${ }^{[42]}$. These functions were also closely related to the occurrence of tumors. The results of KEGG analysis showed that many pathways in these gene-enriched pathways were closely related to cancer progression, such as pathways in cancer, Ras signaling pathway ${ }^{[43]}$, PI3K-Akt signaling pathway ${ }^{[44]}$. It shows that these DEGs can regulate the occurrence, development, invasion and metastasis of breast cancer through a variety of signaling pathways. We constructed a PPI network in the STRING database, and used the MCC algorithm in Cytoscape to get the top ten hub genes. Then we used UALCAN and ONCOMINE database to verify the relative expression levels of these hub genes in breast cancer tissues and found that all the hub genes were dysregulated in breast cancer tissue and the result was consistent. HPA database was used to verify the protein expression level of hub genes. We found statistically different results in the protein expression level of GNG2, ADCY5, CX3CL1, CXCL11, S1PR1. Therefore, female CHB patients may affect the occurrence and development of breast cancer by regulating the expression of miRNAs in exosomes to manipulate the expression of hub genes in breast tissue. 
Chemokines were secreted proteins with a molecular weight of $8-14 \mathrm{kDa}$ in the cytokine family ${ }^{[45]}$. Both immune and tumor cells can express multiple chemokines. According to the number of conserved cysteines in the $\mathrm{N}$-terminal domain of these proteins, they were divided into four families: CC, CXC, CX3C and XC families ${ }^{[46]}$. Chemokines played an important role in tumorigenesis and development. CX3CL1 was a chemokine with a cysteine signature motif -Cys$X-X-X-C y s a t$ the N-terminal end ${ }^{[47]}$ named neurotactin ${ }^{[48]}$ or fractalkine ${ }^{[49]}$ which was highly expressed in brain ${ }^{[50]}$, blood vessel ${ }^{[51]}$, bone ${ }^{[52]}$ and other tissues. Increased expression of CX3CL1 in tumor tissues will cause anti-cancer CD4 + T cells, CD8 + T cells, dendritic cells and NK cells to accumulate around the tumor to kill tumor cells ${ }^{[53,54]}$. High expression of CX3CL1 in breast cancer ${ }^{[55]}$, gastrointestinal cancer ${ }^{[56]}$, lung adenocarcinoma ${ }^{[57]}$ was closely related to good prognosis. On the contrary, advanced tumors will secrete TGF- $\beta$ to reduce the expression of intracellular CX3CL ${ }^{[58]}$. It will inhibit the aggregation of anti-tumor cells to tumor tissues and promote tumor development. By bioinformatics analysis, we found that miR-885-5p was highly expressed in serum exosomes of female CHB patients which would inhibit CX3CL1 expression in breast cells. And the lower expression of CX3CL1 was correlated with higher lymph node metastasis rate in breast cancer patients. Patients with higher lymph node metastasis rate had significantly worse prognosis ${ }^{[59]}$, which may be associated with a worse prognosis for patients with lower expression of CX3CL1. CXCL12 was originally considered to be a growth factor of pre-B cells and was closely related to lymphocyte survival and embryonic development ${ }^{[60]}$. At present, a large number of basic studies had found that CXCL12 promoted the occurrence and development of multiple tumors, invasion and metastasis ${ }^{[61,62]}$ and resistance to chemotherapeutics ${ }^{[63,64]}$ through multiple signaling pathways by combining with $\mathrm{CXCR} 4^{[65,66]}$. And some clinical studies had found that breast cancer patients with high expression of CXCL12 had a poor prognosis ${ }^{[67,68]}$. But there were many basics ${ }^{[69]}$, clinical researches and meta-analysis ${ }^{[70,71]}$ found that high expression of CXCL12 will prolong the survival of patients. By bioinformatics analysis, we found that high expression of miR-125b-5p and miR-1260a in serum exosomes suppressed CXCL12 expression in breast tissue. The bc-GenExMiner database showed that low CXCL12 expression was positively correlated with HER-2 positive status. HER-2 positive status was associated with poor prognosis ${ }^{[72]}$. This may be related to the poor prognosis of patients with low CXCL12 expression in the K-M plotter database. In addition, the main metastatic feature of CXCL12 comes from the biological functions of CXCR4. By inhibiting the expression of CXCR4, the effect of breast cancer immunotherapy was enhanced ${ }^{[73]}$. We searched CXCR4 expression of breast cancer in UALCAN and K-M plotter found that CXCR4 expression was highly expressed in breast cancer tissues(Fig. 5), and the RFS was poor when the expression of CXCR4 was low(Fig. 8). This result indicated that it may not be the increase in endogenous CXCL12 expression, but the high expression of CXCR4 caused by other reasons, that leads to the poor prognosis of breast cancer patients. This had also been confirmed by some researches that decreasing expression of endogenous CXCL12 will enhance the expression of CXCR4 to accept external CXCL12 and enhance mitotic ability ${ }^{[69]}$. Interestingly, we also observed that miR-1237-3p which can inhibit CXCL12 expression was lowly expressed in the serum exosomes of female CHB patients. Whether this change will affect the overall expression of CXCL12 in breast tissue needs to be further investigated. CXCL1, a small chemokine belonging to the $\mathrm{CXC}$ chemokine family, was closely related to inflammation ${ }^{[74]}$, angiogenesis ${ }^{[75]}$, tumor formation ${ }^{[76]}$ and so on. CXCL1 can promote the invasion and metastasis of breast cancer cells by activating ERK to promote the expression of MMP2/9 ${ }^{[77]}$. Breast cancer cells promote CXCL1/S100A8/9 survival axis through high expression of TNF-a to promote breast cancer cells to resist chemotherapy ${ }^{[78]}$. In addition, a large number of clinical studies have found that high expression of CXCL1 was associated with the poor prognosis of a large number of tumors ${ }^{[79]}$. However, we have obtained the opposite result in breast cancer through bioinformatics technology. In this research, we found that high expression of miR-885-5p in serum exosomes of female CHB patients suppressed CXCL1 expression in breast tissue. The low expression of CXCL1 in breast cancer tissue was positively correlated with poor prognosis of breast cancer. Some researchers had found that the expression of CXCL1 in breast cancer 
tissue specimens was significantly lower than that in normal tissues ${ }^{[80]}$. Unfortunately, they had not made a reasonable explanation for this. This conflict may require further research. CXCL11 was induced by IFN- $\gamma$, IFN- $\beta$ and IFN-a ${ }^{[81]}$, mainly through the combination of $\mathrm{CXCR}^{[82]}$ or $\mathrm{CXCR7}$ to play biological function ${ }^{[83]}$. CXCL11 promoted the self-renewal ability, tumorigenicity and anti-chemotherapeutic ability of liver cancer cells through the CXCR3/ERK1/2 signaling pathway ${ }^{[84]}$. Inhibition of CXCL11 expression can inhibit tumor cell proliferation ${ }^{[85]}$ and epithelialmesenchymal transition ${ }^{[86]}$. Our results found that compared with the expression level of miR-363-3p in serum exsomes of normal women, the expression of miR-363-3p was lower in serum exosomes of female CHB patients. This difference will lead to high expression of CXCL11 in breast tissue. High expression of CXCL11 was positively associated with nodal status rate, which may predict a poor prognosis. In addition we obtained similar results in the K-M plotter database, that high expression of CXCL11 was associated with poor prognosis of breast cancer patinets.

Current studies have found that heterotrimers $\mathrm{G}$ proteins were involved in various biological activities such as cell proliferation, differentiation ${ }^{[87]}$ and angiogenesis ${ }^{[88]}$. G protein g2 subunit ( GNG2) was one of the subunits of $\mathrm{G}$ protein $\beta$ dimer. It was expressed in fetal tissue, adrenal gland and brain. It regulated a series of intracellular effects, including ion channels and adenylate cyclase ${ }^{[89]}$ by activating Phospholipase $\mathrm{c} 2$ ( $\left.\mathrm{PlcH} 2\right)$ neurotransmitter deliver ${ }^{[90]}$. Reducing the expression of GNG2 can activate c-SRC and promote AKT signaling pathway, which were closely related to breast cancer cell proliferation ${ }^{[91]}$, invasion and metastasis ${ }^{[92]}$. Our study found that elevated miR-125b-5p expression in serum exosomes of female CHB patients may lead to the low expression of GNG2 in breast cancer tissue, and breast cancer patients with low expression of GNG2 will lead to a poor prognosis.

LPAR1 was the earliest discovered lysophosphatidic acid receptor ${ }^{[93]}$ which was expressed in brain, heart, colon, small intestine and placenta ${ }^{[94]}$. In combination with Gai/o,Gaq/11 and Ga12/13, LPAR1 can activate AKT, rho, MAPK and PLC to affect cell survival, differentiation, adhesion and migration ${ }^{[95]}$. The role of LPAR1 in breast cancer was still unclear. Some studies had found that LPAR1 inhibitor can inhibit breast cancer cell metastasis to the lung and liver ${ }^{[96]}$. Nevertheless, other studies had found that inhibiting LPAR1 expression can promote the proliferation, invasion and metastasis of breast cancer cells ${ }^{[97]}$. Our study found that elevated miR-125b-5p expression in serum exosomes of female CHB patients may lead to LPAR1 down-regulated in breast cancer tissues. Low expression of LPAR1 was positively associated with HER-2 positive status in breast cacaer. HER-2 positive status was associated with poor prognosis ${ }^{[72]}$, which may explain patients with low expression of LPAR1 had poor prognosis. However, we also observed that miR-1237-3p, which can inhibit LPAR1 expression, was decreased in serum exosomes of such patients. Whether this change will affect the expression of LPAR1 in breast cancer needs to be confirmed by more studies.

Protein Coupled Receptors (GPCR) were the unique family of transmembrane receptors and interaction with a diversity set of ligands such as lips, pets, chemokines, odorants, homones and pheromones to achieve its function ${ }^{\text {[98] }}$. At present, it was found that GPR17 was mainly confined to oligodendrocyte cell lines and was very important for myelin formation in oligodendrocytes ${ }^{[99]}$. It was considered to be closely related to multiple sclerosis ${ }^{[100]}$. GPR17 agonists can inhibit glioma cell proliferation ${ }^{[101]}$. At present, there was still no relevant research about GPR17 in breast cancer. According to our results, the expression of GPR17 in breast cancer was reduced by the high expression of miR-1260a, miR-1287-5p and miR-885-5p in serum exosomes of female CHB patients. And the low expression of the gene was correlated with the poor prognosis. This was similar to the results of GPR17 in glioma but it needs to be further confirmed in breast cancer. We also observed that miR-1237-3p and miR-363-3p, which inhibited the expression of GPR17, were lowly expressed in serum exosomes of female CHB patients. More basic and clinical studies should be undertaked to confirme whether this change affected GPR17 expression in breast cancer.

Page 19/34 
Adenylate cyclase can convert ATP into the second messenger cAMP, and influence sugar, lipid and protein metabolism through signal transmission. ADCY5 belongs to the adenylate cyclase family and consists of twelve transmembrane domains, which catalyze the conversion of ATP. It was a cyclic AMP, which was the second messenger of $G$ protein-coupled receptors ${ }^{[102]}$. The low expression of ACDY 5 after DNA methylation was related to the low prognosis of lung cancer ${ }^{[103]}$. The decrease of ACDY5 promoted the occurence of prostate cancer ${ }^{[104]}$. Using bioinformatics technology, we found that highly expressed miR-1260a in serum exosomes of female CHB patients will suppress ADCY5 expression in breast tissue and low expression of ACDY 5 was associated with a poor prognosis. At present, the relationship between ADCY 5 and malignant tumors was not clear. Studies have found that ADCY 5 decline can promote mouse cell autophagy to promote mouse cell survival and resist apoptosis ${ }^{[105]}$, whether this can explain the relationship between ADCY 5 decline and the malignancy remained to be further research. ADCY 3 was widely expressed in tissues throughout the body, and its role in tumors had not yet been determined. Studies had found that its high expression can promote the occurrence and development of gastric cancer through overexpression of $\mathrm{CREB}^{[106]}$, and inhibiting $A D C Y 3$ expression by miR-335 can inhibit gastric cancer cells invasion and metastasis ${ }^{[107]}$. However, other studies had found that promoting the expression of ADCY3 can inhibit the invasion and metastasis of pancreatic cancer ${ }^{[108]}$. Through bioinformatics technology, researchers found that the expression of $A D C Y 3$ in malignant glioma was lower than that in normal tissues, and the low expression of ADCY3 was related to the poor prognosis ${ }^{[109]}$. Our research speculated that female $\mathrm{CHB}$ patients may inhibit the expression of ADCY 3 in breast tissue through high expression of miR-125b-5p in serum exosomes. This alteration would promote breast cancer and was positively correlated with HER-2 positive status. This change can explain the association of low expression of $A D C Y 3$ with poor prognosis in the K-M plotter.

S1P receptor (S1PR1) was a biologically active sphingolipid metabolite that mediated S1P activity and promoted cell proliferation and survival ${ }^{[110,111]}$. S1PR1 was widely expressed in vascular endothelium and it was very important for embryonic blood vessel formation and maturation ${ }^{[112]}$. The role of S1PR1 in tumors was unclear currently. Some studies had found that S1PR1 and STAT3 stimulated and activated each other to enhance tumor growth ${ }^{[113]}$. In addition, S1PR1 can also promote tumor development by activating multiple signaling pathways ${ }^{[114-116]}$. S1PR1 can promote breast cancer cell survival by down-regulating the pro-apoptotic protein Bim and up-regulating the antiapoptotic protein Mcl-1 ${ }^{[17]}$. S1PR1 inhibitors can enhance the toxicity and anti-proliferative effects of carboplatin on breast cancer cells ${ }^{[118]}$. However, some researchers had found that inhibiting the expression of S1PR1 can promote breast cancer and improve survival ${ }^{[119]}$. S1PR1 regulates the activation of RhoA and inhibits the mimic angiogenesis of breast cancer cells to inhibit tumor growth and distant metastasis ${ }^{[120]}$. Some researchers had found that the expression of S1PR1 in breast cancer tissues was significantly lower than that in normal tissues by integrating TCGA data, and lower expression of S1PR1 indicated a worse prognosis ${ }^{[121]}$. By analyzing relevant data, we also found that miR-1287-5p in serum exosomes of female CHB patients may inhibit the expression of S1PR1 in breast tissue. Low expression of S1PR1 was positively associated with poor prognosis. This is consistent with some other scholars.

By comparing the expression of miRNAs in serum exosomes of chronic HBV-infected female with uninfected female, we clarified the reasons why female with chronic HBV infection were more likely to develop breast cancer and had a worse prognosis. Since the data used for the analysis were obtained from multiple online bioinformatics resources, there may be some bias caused by confounding factors. And these theories need to be confirmed in more basic and clinical research.

\section{Conclusion}


In conclusion, female patients with chronic HBV infection may affect the expression of miRNAs in serum exosomes to regulate the target genes in breast tissue, this mechanism will improve the occurrence, invasion and metastasis of breast cancer and lead to a worse prognosis.

\section{Abbreviations}

HBV: Hepatitis b virus; GEO: Gene expression omnibus; DEGs: Differentially expressed genes; PPI: Protein-protein interaction; BP: Biological processes; KEGG: Kyoto Encyclopedia of Genes and Genomes; CXCL11: C-X-C motif chemokine ligand 11; CXCL12: C-X-C motif chemokine ligand 12; GNG2: G-protein gamma 2 subunit; LPAR1: Lysophosphatidic acid receptor 1; GPR17: G protein-coupled receptor 17; CXCL1: C-X-C motif chemokine ligand 1; ADCY5: Adenylate cyclase 5; ADCY3: Adenylate cyclase 3; CX3CL1: C-X-C motif chemokine ligand 3; S1PR1:

Sphingosine-1-Phosphate Receptor 1; MCDE: Molecular Complex Detection; CXCR4: C-X-C chemokine receptor type 4; CXCR3: C-X-C chemokine receptortype 3; CXCR7: C-X-C chemokine receptor type 7; CREB: cAMP response element binding protein; STAT3: Signal Transducer And Activator Of Transcription 3.

\section{Declarations}

\section{Acknowledgements}

Sincerely thank the GEO and TCGA platforms and the authors who uploaded the original data. In addition. Thanks to all the authors who contributed to this article, and to the publisher for supporting this article.

\section{Authors' contributions}

This article was done in collaboration with all the following authors. LQ and LHS determined the research theme and formulated the main research plan. XWJ and CC analyzed the data, explained the results, and wrote the manuscript. All authors read and approved the final manuscript.

\section{Funding}

Qihang funds of Fujian Medical University (2019QH1157)

\section{Availability of data and materials}

In this study, miRNAs microarray datasets were downloaded from the GEO (http://www.ncbi.nlm.nih.gov/geo) and mRNA datasets were downloaded from GEPIA V2.0 database(Gene Expression Profiling Interactive Analysis; http://gepia.cancerpku.cn/) .

\section{Ethics approval and consent to participate}

None

\section{Consent for publication}

Not applicable.

\section{Competing interests}

The authors declare no conflict of interests. 


\section{Author details}

${ }^{1}$ Department of Genertal Surgery, Fujian Provincal Hospital, No. 131 Dongjie Street, Fuzhou, Fujian 350001, People's Republic of China. ${ }^{2}$ Department of Medicine, Fujian Medical University, No. 1 Xuefu Street, Fuzhou, Fujian 350001, People's Republic of China. 3.

\section{References}

1. Shiffman ML. Approach to the patient with chronic hepatitis B and decompensated cirrhosis. Liver Int. 2020. 40 Suppl 1: 22-26.

2. Xie M, Guo H, Lou G, et al. Neddylation inhibitor MLN4924 has anti-HBV activity via modulating the ERK-HNF1aC/EBPa-HNF4a axis. J Cell Mol Med. 2021. 25(2): 840-854.

3. An J, Kim JW, Shim JH, et al. Chronic hepatitis B infection and non-hepatocellular cancers: A hospital registrybased, case-control study. PLoS One. 2018. 13(3): e0193232.

4. Siegel RL, Miller KD, Jemal A. Cancer statistics, 2020. CA Cancer J Clin. 2020. 70(1): 7-30.

5. Lu T, Yang Q, Li M, et al. HBV infection and extra-hepatic cancers in adolescents and 20s: A retrospective study in China. Cancer Epidemiol. 2018. 55: 149-155.

6. Xiao $W, Z$ hou $Y, Y u$, et al. Prognostic value of chronic hepatitis $B$ virus infection in patients with breast cancer in a hepatitis $B$ virus endemic area. Ann Transl Med. 2020. 8(5): 180.

7. Makvandi M. Update on occult hepatitis B virus infection. World J Gastroenterol. 2016. 22(39): 8720-8734.

8. Ziegler RG, Fuhrman BJ, Moore SC, Matthews CE. Epidemiologic studies of estrogen metabolism and breast cancer. Steroids. 2015. 99(Pt A): 67-75.

9. Qin B, Zhao K, Wei J, et al. Novel evidence indicates the presence and replication of hepatitis $B$ virus in breast cancer tissue. Oncol Rep. 2020. 43(1): 296-305.

10. Schubert D. A Brief History of Adherons: The Discovery of Brain Exosomes. Int J Mol Sci. 2020. 21(20).

11. Tkach M, Théry C. Communication by Extracellular Vesicles: Where We Are and Where We Need to Go. Cell. 2016. 164(6): 1226-1232.

12. Fu M, Gu J, Jiang P, Qian H, Xu W, Zhang X. Exosomes in gastric cancer: roles, mechanisms, and applications. Mol Cancer. 2019. 18(1): 41.

13. Jalalian SH, Ramezani M, Jalalian SA, Abnous K, Taghdisi SM. Exosomes, new biomarkers in early cancer detection. Anal Biochem. 2019. 571: 1-13.

14. Yagi Y, Ohkubo T, Kawaji H, et al. Next-generation sequencing-based small RNA profiling of cerebrospinal fluid exosomes. Neurosci Lett. 2017. 636: 48-57.

15. Qin W, Tsukasaki Y, Dasgupta S, Mukhopadhyay N, Ikebe M, Sauter ER. Exosomes in Human Breast Milk Promote EMT. Clin Cancer Res. 2016. 22(17): 4517-24.

16. van der Pol E, Böing AN, Harrison P, Sturk A, Nieuwland R. Classification, functions, and clinical relevance of extracellular vesicles. Pharmacol Rev. 2012. 64(3): 676-705.

17. Melo SA, Luecke LB, Kahlert C, et al. Glypican-1 identifies cancer exosomes and detects early pancreatic cancer. Nature. 2015. 523(7559): $177-82$.

18. Dong X, Bai X, Ni J, et al. Exosomes and breast cancer drug resistance. Cell Death Dis. 2020. 11(11): 987.

19. Giordano C, La Camera G, Gelsomino L, et al. The Biology of Exosomes in Breast Cancer Progression: Dissemination, Immune Evasion and Metastatic Colonization. Cancers (Basel). 2020. 12(8).

Page 22/34 
20. Lak R, Yaghobi R, Garshasbi M. Importance of miR-125a-5p and miR-122-5p expression in patients with HBV infection. Cell Mol Biol (Noisy-le-grand). 2020. 66(5): 1-8.

21. Ma S, Qin K, Ouyang H, Zhu H, Lei P, Shen G. HBV infection exacerbates PTEN defects in hepatocellular carcinoma through upregulation of miR-181a/382/362/19a. Am J Transl Res. 2020. 12(7): 3780-3791.

22. Guyon N, Garnier D, Briand J, et al. Anti-PD1 therapy induces lymphocyte-derived exosomal miRNA-4315 release inhibiting Bim-mediated apoptosis of tumor cells. Cell Death Dis. 2020. 11(12): 1048.

23. Murakami Y, Toyoda H, Tanahashi T, et al. Comprehensive miRNA expression analysis in peripheral blood can diagnose liver disease. PLoS One. 2012. 7(10): e48366.

24. Sticht C, De La Torre C, Parveen A, Gretz N. miRWalk: An online resource for prediction of microRNA binding sites. PLoS One. 2018. 13(10): e0206239.

25. Tang Z, Li C, Kang B, Gao G, Li C, Zhang Z. GEPIA: a web server for cancer and normal gene expression profiling and interactive analyses. Nucleic Acids Res. 2017. 45(W1): W98-W102.

26. Chen EY, Tan CM, Kou Y, et al. Enrichr: interactive and collaborative HTML5 gene list enrichment analysis tool. BMC Bioinformatics. 2013. 14: 128.

27. Kuleshov MV, Jones MR, Rouillard AD, et al. Enrichr: a comprehensive gene set enrichment analysis web server 2016 update. Nucleic Acids Res. 2016. 44(W1): W90-7.

28. Chandrashekar DS, Bashel B, Balasubramanya S, et al. UALCAN: A Portal for Facilitating Tumor Subgroup Gene Expression and Survival Analyses. Neoplasia. 2017. 19(8): 649-658.

29. Rhodes DR, Kalyana-Sundaram S, Mahavisno V, et al. Oncomine 3.0: genes, pathways, and networks in a collection of 18,000 cancer gene expression profiles. Neoplasia. 2007. 9(2): $166-80$.

30. Uhlén M, Fagerberg L, Hallström BM, et al. Proteomics. Tissue-based map of the human proteome. Science. 2015. 347(6220): 1260419.

31. Jézéquel P, Gouraud W, Ben Azzouz F, et al. bc-GenExMiner 4.5: new mining module computes breast cancer differential gene expression analyses. Database (Oxford). 2021. 2021.

32. Nagy Á, Lánczky A, Menyhárt O, Györffy B. Validation of miRNA prognostic power in hepatocellular carcinoma using expression data of independent datasets. Sci Rep. 2018. 8(1): 9227.

33. Huang J, Chan PS, Lok V, et al. Global incidence and mortality of breast cancer: a trend analysis. Aging (Albany NY). 2021. 13(4): 5748-5803.

34. Li N, Zhong QQ, Yang XR, et al. Prognostic Value of Hepatitis B Virus Infection in Very Young Patients With Curatively Resected Breast Cancer: Analyses From an Endemic Area in China. Front Oncol. 2020. 10: 1403.

35. Clucas J, Valderrama F. ERM proteins in cancer progression. J Cell Sci. 2015. 128(6): 1253.

36. Sulzmaier FJ, Jean C, Schlaepfer DD. FAK in cancer: mechanistic findings and clinical applications. Nat Rev Cancer. 2014. 14(9): 598-610.

37. Al Absi A, Wurzer H, Guerin C, et al. Actin Cytoskeleton Remodeling Drives Breast Cancer Cell Escape from Natural Killer-Mediated Cytotoxicity. Cancer Res. 2018. 78(19): 5631-5643.

38. Mueller C, Haymond A, Davis JB, Williams A, Espina V. Protein biomarkers for subtyping breast cancer and implications for future research. Expert Rev Proteomics. 2018. 15(2): 131-152.

39. Cenciarini ME, Proietti CJ. Molecular mechanisms underlying progesterone receptor action in breast cancer: Insights into cell proliferation and stem cell regulation. Steroids. 2019. 152: 108503.

40. Izdebska M, Zielińska W, Grzanka D, Gagat M. The Role of Actin Dynamics and Actin-Binding Proteins Expression in Epithelial-to-Mesenchymal Transition and Its Association with Cancer Progression and Evaluation of Possible 
Therapeutic Targets. Biomed Res Int. 2018. 2018: 4578373.

41. Steigedal TS, Toraskar J, Redvers RP, et al. Nephronectin is Correlated with Poor Prognosis in Breast Cancer and Promotes Metastasis via its Integrin-Binding Motifs. Neoplasia. 2018. 20(4): 387-400.

42. Yang W, Hosford SR, Traphagen NA, et al. Autophagy promotes escape from phosphatidylinositol 3-kinase inhibition in estrogen receptor-positive breast cancer. FASEB J. 2018. 32(3): 1222-1235.

43. Wright KL, Adams JR, Liu JC, et al. Ras Signaling Is a Key Determinant for Metastatic Dissemination and Poor Survival of Luminal Breast Cancer Patients. Cancer Res. 2015. 75(22): 4960-72.

44. Nunnery SE, Mayer IA. Targeting the PI3K/AKT/mTOR Pathway in Hormone-Positive Breast Cancer. Drugs. 2020. 80(16): 1685-1697.

45. Kazanietz MG, Durando M, Cooke M. CXCL 13 and Its Receptor CXCR5 in Cancer: Inflammation, Immune Response, and Beyond. Front Endocrinol (Lausanne). 2019. 10: 471.

46. Hughes CE, Nibbs R. A guide to chemokines and their receptors. FEBS J. 2018. 285(16): 2944-2971.

47. Bazan JF, Bacon KB, Hardiman G, et al. A new class of membrane-bound chemokine with a CX3C motif. Nature. 1997. 385(6617): 640-4.

48. Pan Y, Lloyd C, Zhou H, et al. Neurotactin, a membrane-anchored chemokine upregulated in brain inflammation. Nature. 1997. 387(6633): 611-7.

49. Imai T, Hieshima K, Haskell C, et al. Identification and molecular characterization of fractalkine receptor CX3CR1, which mediates both leukocyte migration and adhesion. Cell. 1997. 91(4): 521 - 30.

50. Noda M, Doi Y, Liang J, et al. Fractalkine attenuates excito-neurotoxicity via microglial clearance of damaged neurons and antioxidant enzyme heme oxygenase-1 expression. J Biol Chem. 2011. 286(3): 2308-19.

51. Sidibe A, Ropraz P, Jemelin $S$, et al. Angiogenic factor-driven inflammation promotes extravasation of human proangiogenic monocytes to tumours. Nat Commun. 2018. 9(1): 355.

52. Liang Y, Yi L, Liu P, et al. CX3CL 1 involves in breast cancer metastasizing to the spine via the SrC/FAK signaling pathway. J Cancer. 2018. 9(19): 3603-3612.

53. Yu YR, Fong AM, Combadiere C, Gao JL, Murphy PM, Patel DD. Defective antitumor responses in CX3CR1deficient mice. Int J Cancer. 2007. 121(2): 316 - 22.

54. Guo J, Chen T, Wang B, et al. Chemoattraction, adhesion and activation of natural killer cells are involved in the antitumor immune response induced by fractalkine/CX3CL 1. Immunol Lett. 2003. 89(1): 1-7.

55. Park MH, Lee JS, Yoon JH. High expression of CX3CL 1 by tumor cells correlates with a good prognosis and increased tumor-infiltrating CD8 + T cells, natural killer cells, and dendritic cells in breast carcinoma. J Surg Oncol. 2012. 106(4): $386-92$.

56. Erreni M, Siddiqui I, Marelli G, et al. The Fractalkine-Receptor Axis Improves Human Colorectal Cancer Prognosis by Limiting Tumor Metastatic Dissemination. J Immunol. 2016. 196(2): 902 - 14.

57. LiU J, Li Y, Zhu X, et al. Increased CX3CL $1 \mathrm{mRNA}$ expression level is a positive prognostic factor in patients with lung adenocarcinoma. Oncol Lett. 2019. 17(6): 4877-4890.

58. Sciumè G, Soriani A, Piccoli M, Frati L, Santoni A, Bernardini G. CX3CR1/CX3CL 1 axis negatively controls glioma cell invasion and is modulated by transforming growth factor- $\beta 1$. Neuro Oncol. 2010. 12(7): $701-10$.

59. Wen J, Yang Y, Liu P, et al. Development and validation of a nomogram for predicting survival on the base of modified lymph node ratio in breast cancer patients. Breast. 2017. 33: 14-22.

60. Nagasawa T, Hirota S, Tachibana K, et al. Defects of B-cell lymphopoiesis and bone-marrow myelopoiesis in mice lacking the CXC chemokine PBSF/SDF-1. Nature. 1996. 382(6592): 635-8. 
61. Maroni P, Bendinelli P, Matteucci E, Desiderio MA. HGF induces CXCR4 and CXCL 12-mediated tumor invasion through Ets1 and NF-kappaB. Carcinogenesis. 2007. 28(2): 267 - 79.

62. Yu T, Wu Y, Helman JI, Wen Y, Wang C, Li L. CXCR4 promotes oral squamous cell carcinoma migration and invasion through inducing expression of MMP-9 and MMP-13 via the ERK signaling pathway. Mol Cancer Res. 2011. 9(2): $161-72$.

63. Nakamura T, Shinriki S, Jono H, et al. Intrinsic TGF- 2 2-triggered SDF-1-CXCR4 signaling axis is crucial for drug resistance and a slow-cycling state in bone marrow-disseminated tumor cells. Oncotarget. 2015. 6(2): $1008-19$.

64. Dubrovska A, Hartung A, Bouchez LC, et al. CXCR4 activation maintains a stem cell population in tamoxifenresistant breast cancer cells through AhR signalling. Br J Cancer. 2012. 107(1): 43-52.

65. Jiang C, Ma S, Hu R, et al. Effect of CXCR4 on Apoptosis in Osteosarcoma Cells via the PI3K/Akt/NF-K $\beta$ Signaling Pathway. Cell Physiol Biochem. 2018. 46(6): 2250-2260.

66. Mirandola L, Apicella L, Colombo M, et al. Anti-Notch treatment prevents multiple myeloma cells localization to the bone marrow via the chemokine system CXCR4/SDF-1. Leukemia. 2013. 27(7): 1558-66.

67. Ramos EA, Camargo AA, Braun K, et al. Simultaneous CXCL 12 and ESR1 CpG island hypermethylation correlates with poor prognosis in sporadic breast cancer. BMC Cancer. 2010. 10: 23.

68. Wu W, Qian L, Chen X, Ding B. Prognostic significance of CXCL 12, CXCR4, and CXCR7 in patients with breast cancer. Int J Clin Exp Pathol. 2015. 8(10): 13217-24.

69. Wendt MK, Cooper AN, Dwinell MB. Epigenetic silencing of CXCL 12 increases the metastatic potential of mammary carcinoma cells. Oncogene. 2008. 27(10): 1461-71.

70. Samarendra H, Jones K, Petrinic T, et al. A meta-analysis of CXCL 12 expression for cancer prognosis. Br J Cancer. 2017. 117(1): 124-135.

71. Liu H, Li Z, Deng M, et al. Prognostic and clinicopathological value of CXCL 12/SDF1 expression in breast cancer: A meta-analysis. Clin Chim Acta. 2018. 484: 72-80.

72. van Maaren $M C$, de Munck $L$, Strobbe $L$, et al. Ten-year recurrence rates for breast cancer subtypes in the Netherlands: A large population-based study. Int J Cancer. 2019. 144(2): 263-272.

73. Pernas S, Martin M, Kaufman PA, et al. Balixafortide plus eribulin in HER2-negative metastatic breast cancer: a phase 1, single-arm, dose-escalation trial. Lancet Oncol. 2018. 19(6): 812-824.

74. Haskill S, Peace A, Morris J, et al. Identification of three related human GRO genes encoding cytokine functions. Proc Natl Acad Sci U S A. 1990. 87(19): 7732-6.

75. Padilla L, Argüero-Sánchez R, Rodríguez-Trejo JM, et al. Effect of autologous transplant of peripheral blood mononuclear cells in combination with proangiogenic factors during experimental revascularization of lower limb ischemia. J Tissue Eng Regen Med. 2020. 14(4): 600-608.

76. Wang YY, Chen HD, Lo S, et al. Visfatin Enhances Breast Cancer Progression through CXCL 1 Induction in TumorAssociated Macrophages. Cancers (Basel). 2020. 12(12).

77. Yang $C, Y u H$, Chen $R$, et al. CXCL 1 stimulates migration and invasion in ER-negative breast cancer cells via activation of the ERK/MMP2/9 signaling axis. Int J Oncol. 2019. 55(3): 684-696.

78. Acharyya S, Oskarsson T, Vanharanta S, et al. A CXCL 1 paracrine network links cancer chemoresistance and metastasis. Cell. 2012. 150(1): 165 - 78.

79. Zhang Z, Chen Y, Jiang Y, Luo Y, Zhang H, Zhan Y. Prognostic and clinicopathological significance of CXCL 1 in cancers: a systematic review and meta-analysis. Cancer Biol Ther. 2019. 20(11): 1380-1388. 
80. Abrahamsson A, Rzepecka A, Dabrosin C. Equal Pro-inflammatory Profiles of CCLs, CXCLs, and Matrix Metalloproteinases in the Extracellular Microenvironment In Vivo in Human Dense Breast Tissue and Breast Cancer. Front Immunol. 2017. 8: 1994.

81. Rani MR, Foster GR, Leung S, Leaman D, Stark GR, Ransohoff RM. Characterization of beta-R1, a gene that is selectively induced by interferon beta (IFN-beta) compared with IFN-alpha. J Biol Chem. 1996. 271(37): 2287884.

82. Colvin RA, Campanella GS, Sun J, Luster AD. Intracellular domains of CXCR3 that mediate CXCL9, CXCL 10, and CXCL 11 function. J Biol Chem. 2004. 279(29): 30219-27.

83. Burns JM, Summers BC, Wang Y, et al. A novel chemokine receptor for SDF-1 and I-TAC involved in cell survival, cell adhesion, and tumor development. J Exp Med. 2006. 203(9): 2201-13.

84. Zhang Y, Zhao W, Li S, et al. CXCL 11 promotes self-renewal and tumorigenicity of a2 1 + liver tumor-initiating cells through CXCR3/ERK1/2 signaling. Cancer Lett. 2019. 449: 163-171.

85. Wang $Y, X u H$, Si L, et al. MiR-206 inhibits proliferation and migration of prostate cancer cells by targeting CXCL 11. Prostate. 2018. 78(7): 479-490.

86. Gao YJ, Liu L, Li S, et al. Down-regulation of CXCL 11 inhibits colorectal cancer cell growth and epithelialmesenchymal transition. Onco Targets Ther. 2018. 11: 7333-7343.

87. Schwindinger WF, Robishaw JD. Heterotrimeric G-protein betagamma-dimers in growth and differentiation. Oncogene. 2001. 20(13): 1653-60.

88. Leung $T$, Chen $H$, Stauffer AM, et al. Zebrafish $G$ protein gamma2 is required for VEGF signaling during angiogenesis. Blood. 2006. 108(1): 160-6.

89. Modarressi MH, Taylor KE, Wolfe J. Cloning, characterization, and mapping of the gene encoding the human $G$ protein gamma 2 subunit. Biochem Biophys Res Commun. 2000. 272(2): 610-5.

90. Zhou Y, Wing MR, Sondek J, Harden TK. Molecular cloning and characterization of PLC-eta2. Biochem J. 2005. 391(Pt 3): $667-76$.

91. Hiscox S, Morgan L, Green T, Nicholson RI. Src as a therapeutic target in anti-hormone/anti-growth factorresistant breast cancer. Endocr Relat Cancer. 2006. 13 Suppl 1: S53-9.

92. Zhang L, Qin Y, Wu G, et al. PRRG4 promotes breast cancer metastasis through the recruitment of NEDD4 and downregulation of Robo1. Oncogene. 2020. 39(49): 7196-7208.

93. Hecht JH, Weiner JA, Post SR, Chun J. Ventricular zone gene-1 (vzg-1) encodes a lysophosphatidic acid receptor expressed in neurogenic regions of the developing cerebral cortex. J Cell Biol. 1996. 135(4): 1071-83.

94. Choi JW, Herr DR, Noguchi K, et al. LPA receptors: subtypes and biological actions. Annu Rev Pharmacol Toxicol. 2010. 50: $157-86$.

95. Xiang H, Lu Y, Shao M, Wu T. Lysophosphatidic Acid Receptors: Biochemical and Clinical Implications in Different Diseases. J Cancer. 2020. 11(12): 3519-3535.

96. Marshall JC, Collins JW, Nakayama J, et al. Effect of inhibition of the lysophosphatidic acid receptor 1 on metastasis and metastatic dormancy in breast cancer. J Natl Cancer Inst. 2012. 104(17): 1306-19.

97. Ma X, Feng J, Lu M, et al. microRNA-501-5p promotes cell proliferation and migration in gastric cancer by downregulating LPAR1. J Cell Biochem. 2020. 121(2): 1911-1922.

98. Van Oekelen D, Luyten WH, Leysen JE. Ten years of antisense inhibition of brain G-protein-coupled receptor function. Brain Res Brain Res Rev. 2003. 42(2): 123 - 42. 
99. Chen $Y, W u H$, Wang $S$, et al. The oligodendrocyte-specific $G$ protein-coupled receptor GPR17 is a cell-intrinsic timer of myelination. Nat Neurosci. 2009. 12(11): 1398 - 406.

100. Dziedzic A, Miller E, Saluk-Bijak J, Bijak M. The GPR17 Receptor-A Promising Goal for Therapy and a Potential Marker of the Neurodegenerative Process in Multiple Sclerosis. Int J Mol Sci. 2020. 21(5).

101. Dougherty JD, Fomchenko El, Akuffo AA, et al. Candidate pathways for promoting differentiation or quiescence of oligodendrocyte progenitor-like cells in glioma. Cancer Res. 2012. 72(18): 4856-68.

102. Defer N, Best-Belpomme M, Hanoune J. Tissue specificity and physiological relevance of various isoforms of adenylyl cyclase. Am J Physiol Renal Physiol. 2000. 279(3): F400-16.

103. Sato T, Arai E, Kohno T, et al. DNA methylation profiles at precancerous stages associated with recurrence of lung adenocarcinoma. PLoS One. 2013. 8(3): e59444.

104. Li D, Hao X, Song Y. Identification of the Key MicroRNAs and the miRNA-mRNA Regulatory Pathways in Prostate Cancer by Bioinformatics Methods. Biomed Res Int. 2018. 2018: 6204128.

105. Du WW, Yang W, Fang L, et al. miR-17 extends mouse lifespan by inhibiting senescence signaling mediated by MKP7. Cell Death Dis. 2014. 5(7): e1355.

106. Hong SH, Goh SH, Lee SJ, et al. Upregulation of adenylate cyclase 3 (ADCY3) increases the tumorigenic potential of cells by activating the CREB pathway. Oncotarget. 2013. 4(10): $1791-803$.

107. Zou J, Wu K, Lin C, Jie ZG. LINC00319 acts as a microRNA-335-5p sponge to accelerate tumor growth and metastasis in gastric cancer by upregulating ADCY3. Am J Physiol Gastrointest Liver Physiol. 2020. 318(1): G10G22.

108. Quinn SN, Graves SH, Dains-McGahee C, et al. Adenylyl cyclase 3/adenylyl cyclase-associated protein 1 (CAP1) complex mediates the anti-migratory effect of forskolin in pancreatic cancer cells. Mol Carcinog. 2017. 56(4): 1344-1360.

109. Lu WC, Xie H, Yuan C, Li JJ, Li ZY, Wu AH. Identification of potential biomarkers and candidate small molecule drugs in glioblastoma. Cancer Cell Int. 2020. 20: 419.

110. Spiegel S, Milstien S. The outs and the ins of sphingosine-1-phosphate in immunity. Nat Rev Immunol. 2011. 11(6): $403-15$.

111. Spiegel S, Milstien S. Sphingosine 1-phosphate, a key cell signaling molecule. J Biol Chem. 2002. 277(29): 258514.

112. Proia RL, Hla T. Emerging biology of sphingosine-1-phosphate: its role in pathogenesis and therapy. J Clin Invest. 2015. 125(4): 1379-87.

113. Lee H, Deng J, Kujawski M, et al. STAT3-induced S1PR1 expression is crucial for persistent STAT3 activation in tumors. Nat Med. 2010. 16(12): 1421-8.

114. Paik JH, Nam SJ, Kim TM, Heo DS, Kim CW, Jeon YK. Overexpression of sphingosine-1-phosphate receptor 1 and phospho-signal transducer and activator of transcription 3 is associated with poor prognosis in rituximab-treated diffuse large B-cell lymphomas. BMC Cancer. 2014. 14: 911.

115. Brinkmann V. Sphingosine 1-phosphate receptors in health and disease: mechanistic insights from gene deletion studies and reverse pharmacology. Pharmacol Ther. 2007. 115(1): 84-105.

116. Dong H, Claffey KP, Brocke S, Epstein PM. Inhibition of breast cancer cell migration by activation of cAMP signaling. Breast Cancer Res Treat. 2015. 152(1): 17-28.

117. Rutherford C, Childs S, Ohotski J, et al. Regulation of cell survival by sphingosine-1-phosphate receptor S1P1 via reciprocal ERK-dependent suppression of Bim and Pl-3-kinase/protein kinase C-mediated upregulation of Mcl-1. 
Cell Death Dis. 2013. 4(11): e927.

118. Xiao S, Yang J. Preclinical study of the antitumor effect of sphingosine-1-phosphate receptor 1 antibody (S1PR(1)-antibody) against human breast cancer cells. Invest New Drugs. 2019. 37(1): 57-64.

119. Lei FJ, Cheng BH, Liao PY, et al. Survival benefit of sphingosin-1-phosphate and receptors expressions in breast cancer patients. Cancer Med. 2018. 7(8): 3743-3754.

120. Cartier A, Leigh T, Liu CH, Hla T. Endothelial sphingosine 1-phosphate receptors promote vascular normalization and antitumor therapy. Proc Natl Acad Sci U S A. 2020. 117(6): 3157-3166.

121. Zhong L, Xie L, Yang Z, et al. Prognostic value of S1PR1 and its correlation with immune infiltrates in breast and lung cancers. BMC Cancer. 2020. 20(7): 766.

\section{Figures}

A

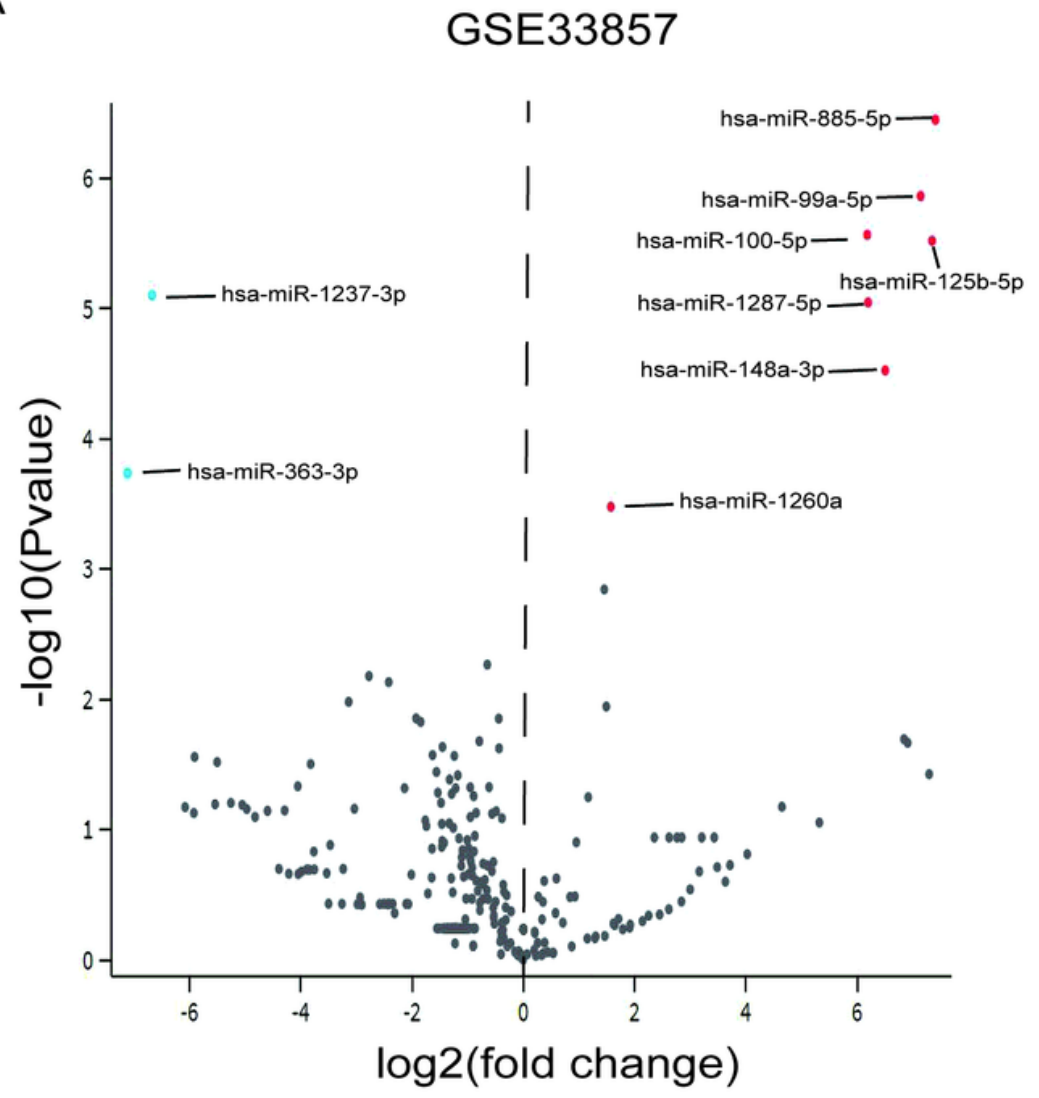

B

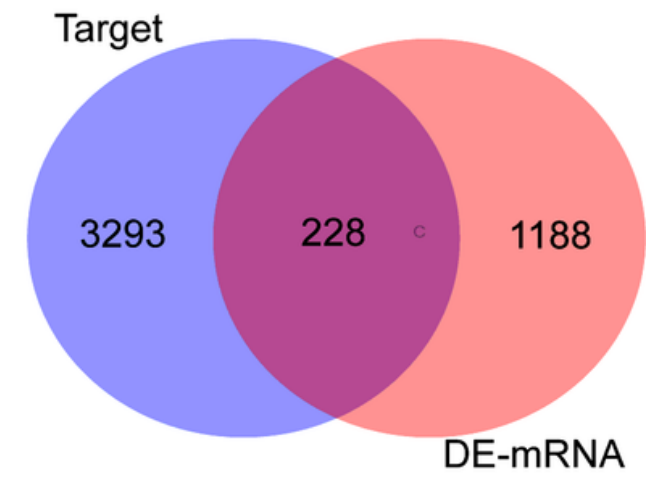

C

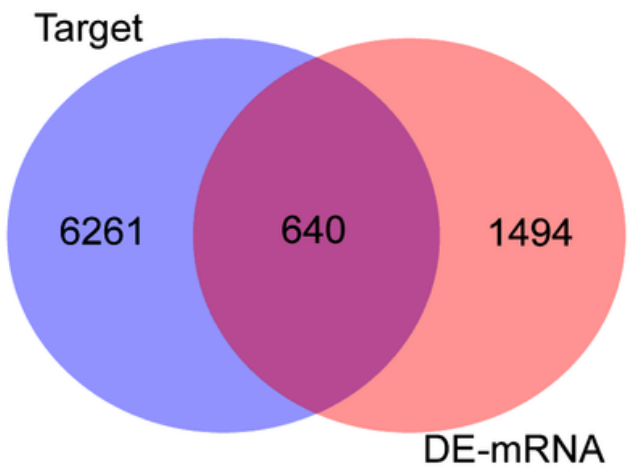

Figure 1

Identification of differentially expressed genes in the expression omnibus (GEO) datasets. (A) Volcano plot of differentially expressed miRNAs in GSE33857. Red and blue color represent relatively high and low expression of miRNAs in the corresponding group, respectively. $p<0.01$ and $|\log (\mathrm{FC})|>1.5$ were set as the threshold. (B-C) Venn diagrams showing the proportion of overlapping genes that were associated to down-regulated miRNAs and showed an increased expression level in breast cancer (C), alongside the proportion of genes that were associated to upregulated miRNAs and showed a decreased expression level (D).Blue-targeted genes of differentially expressed miRNAs; Red-targeted genes of differentially expressed miRNA of breast cancer in GEPIA database. 


\section{Image not available with this version}

\section{Figure 2}

GO analysis and KEGG pathway analysis of DEGs. (A) Top 10 of biological process(BP). (B) Top ten of cellular component(CC). (C) Top ten of molecular function(MF). (D) Top ten of KEGG pathway enrichment

A

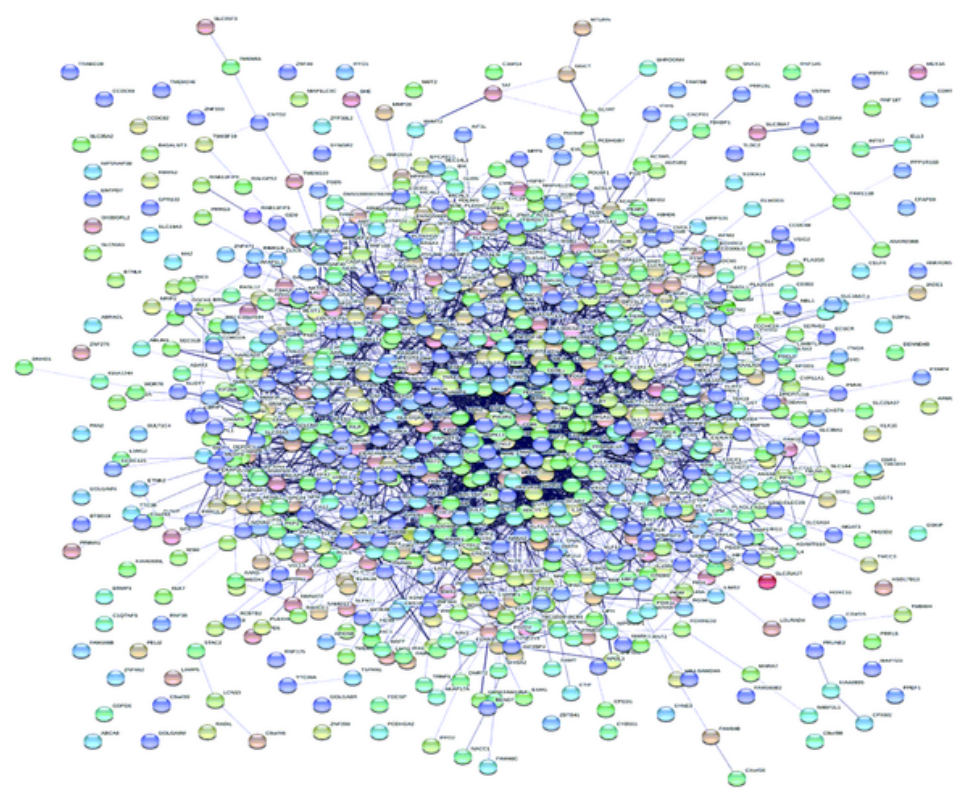

B

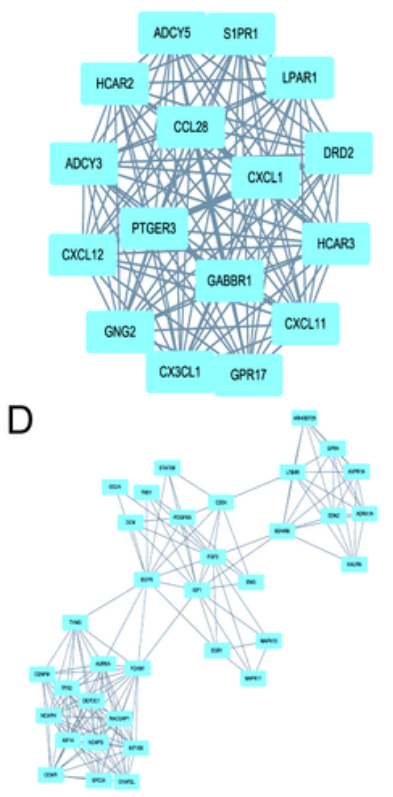

C

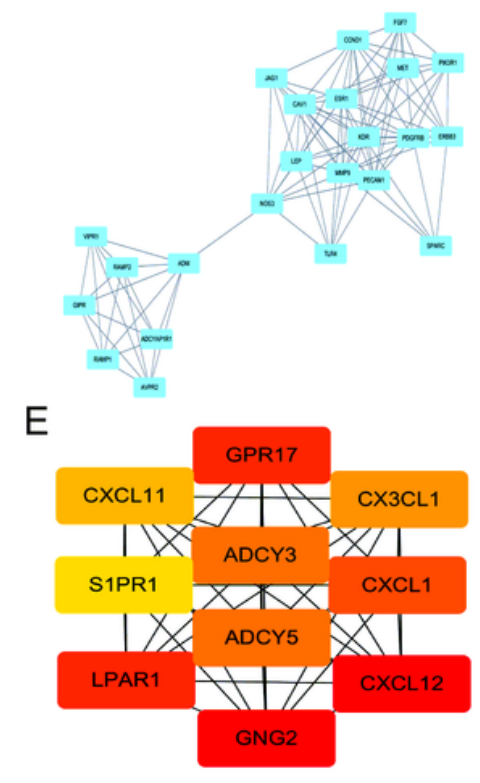

\section{Figure 3}

PPI network, module analysis, and hub genes identification. (A) PPI network of overlapping differentially expressed genes was constructed in STRING database. (B-D) Top three modules screened using MCODE in Cytoscape software. (E) Top ten hub genes selected by the CytoHubba in Cytoscape based on the degree of each protein node. 


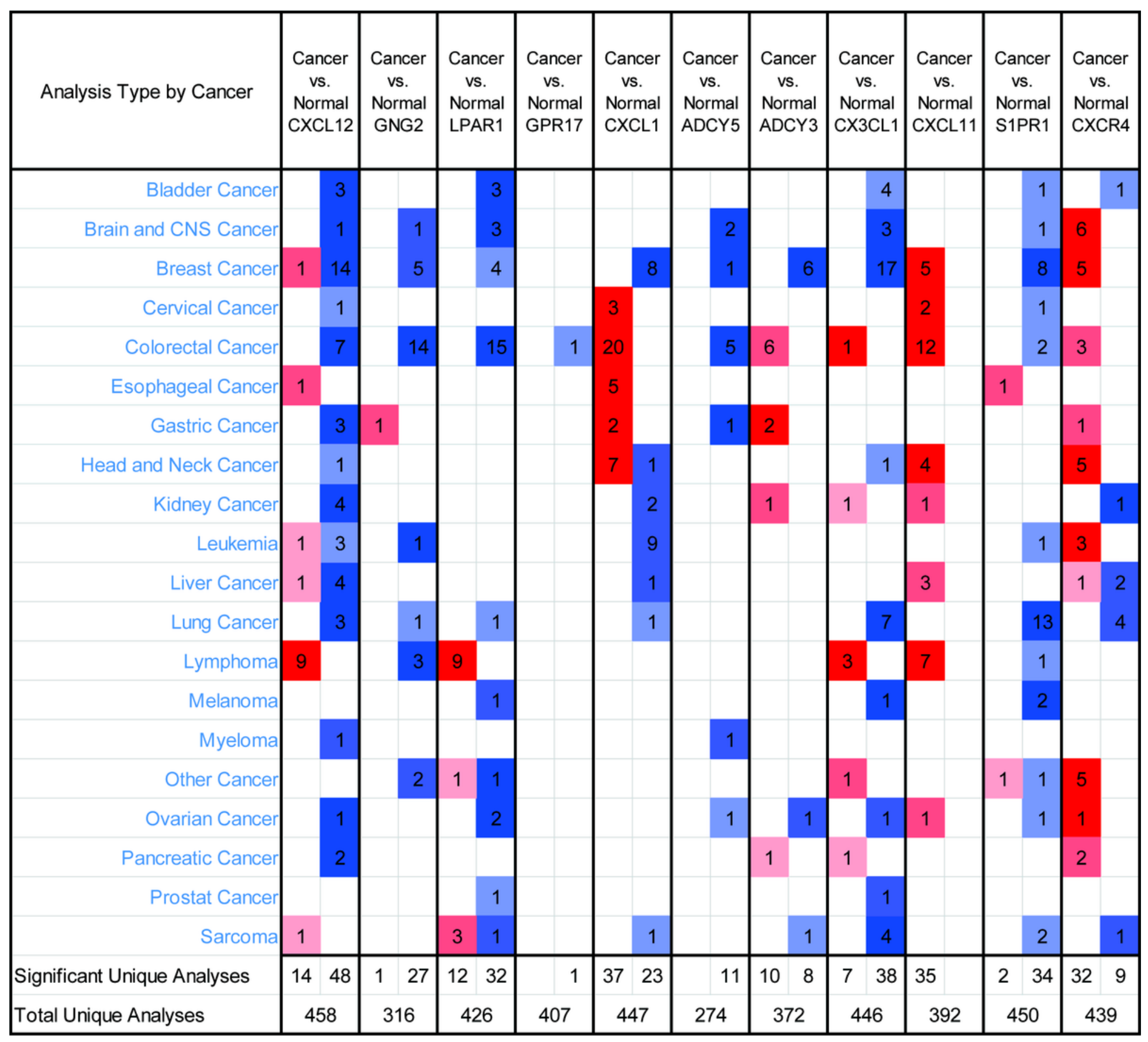

Figure 4

An overview of mRNA levels of hub genes and CXCR4 in a variety of cancers based on Oncomine. The transcription levels of hub genes and CXCR4 in different types of cancer in Oncomine. The numbers in colored cells show the quantities of datasets with statistically significant mRNA overexpression (red) or underexpression (blue) of target genes. Cell color was determined by the best gene rank percentile for the analysis within the cells. The threshold was

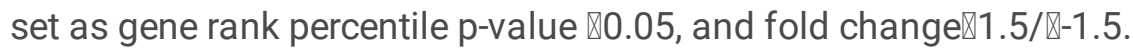



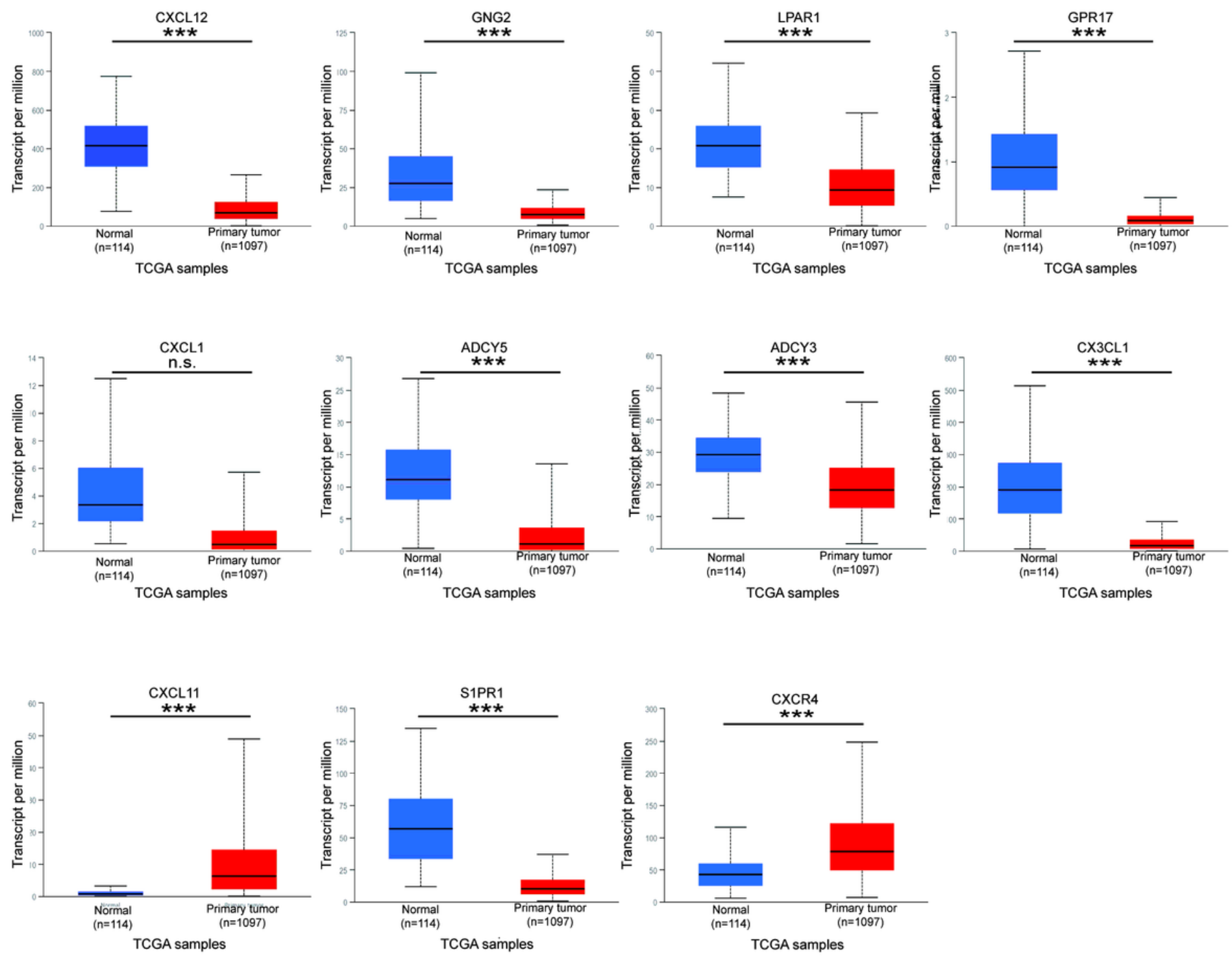

Figure 5

Relative expression of hub genes and CXCR4 in breast cancer samples in UALCAN database. (Student's t-test, ***p》 0.001) Blue: breast cancer sample; Red: control sample. Image adaped from HPA database. 

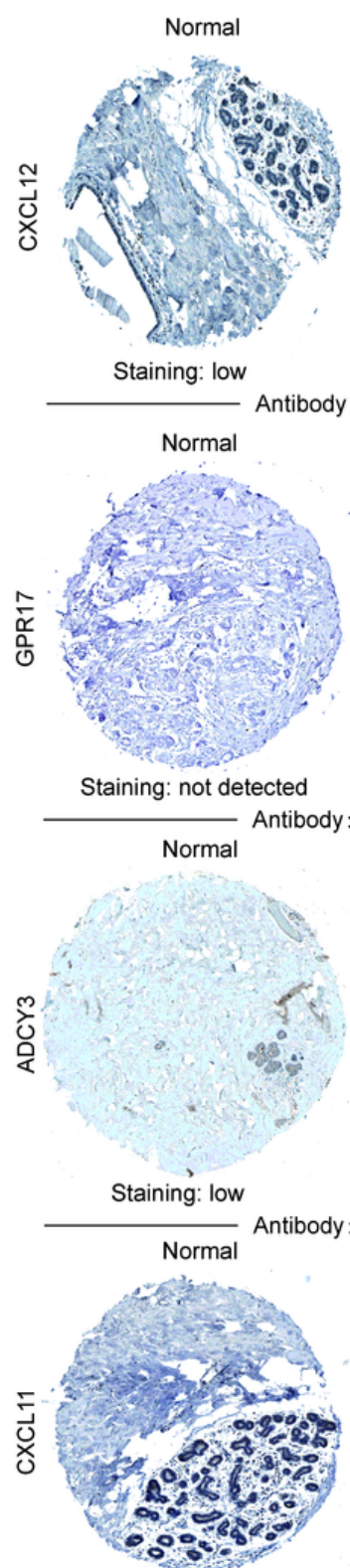

Staining: low

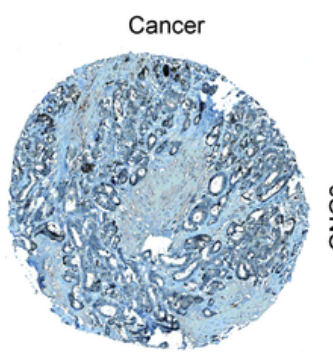

Staining: low
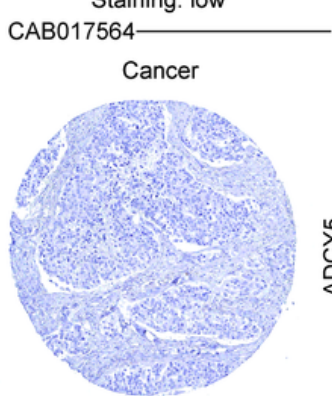

Staining: not detected HPA029766

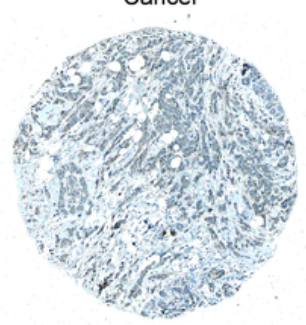

Staining: low

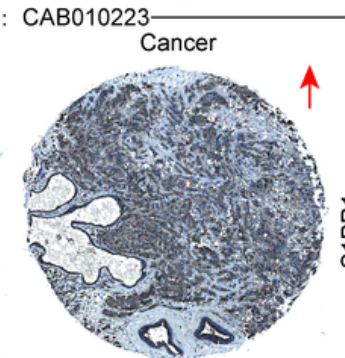

Staining: medium

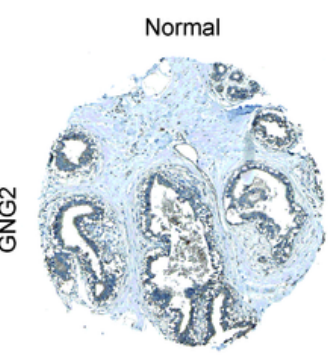

Staining: medium

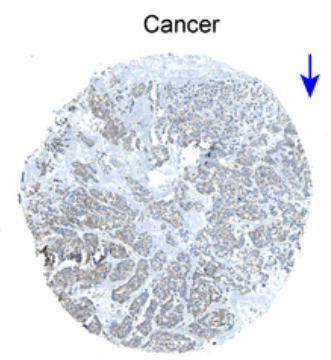

Staining: low

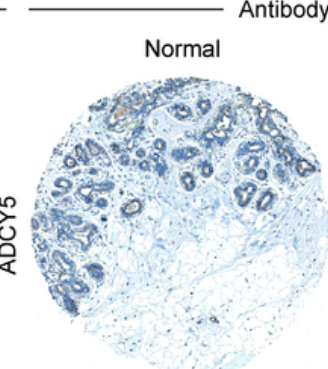

Staining: medium

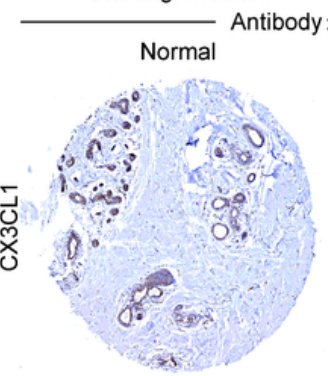

Staining: high

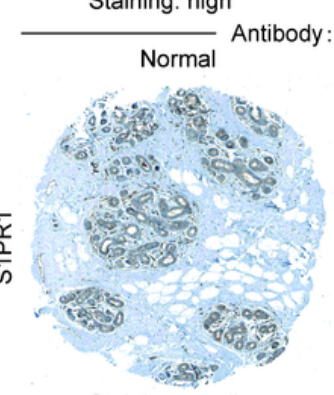

Staining: medium

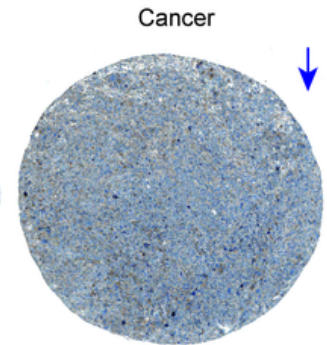

Staining: low
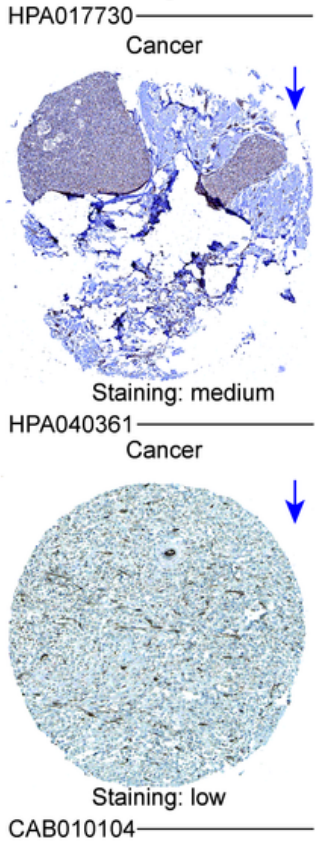

\section{Figure 6}

A caption was not provided with this version 


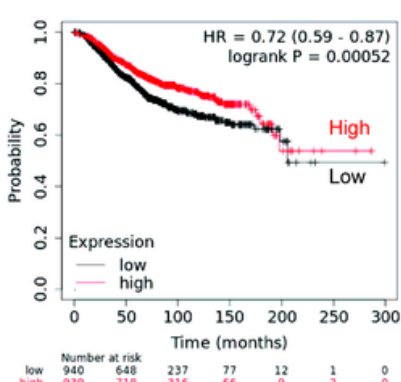

CXCL12

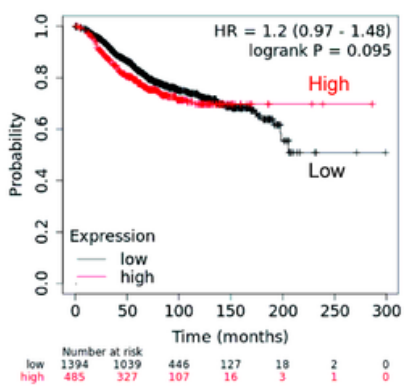

CXCL1

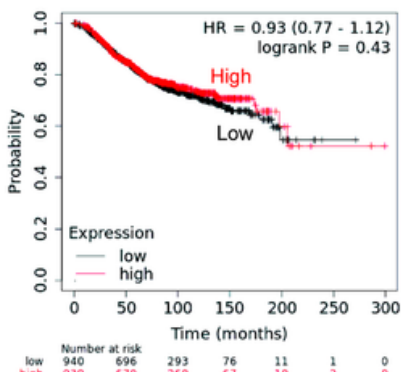

CXCL11

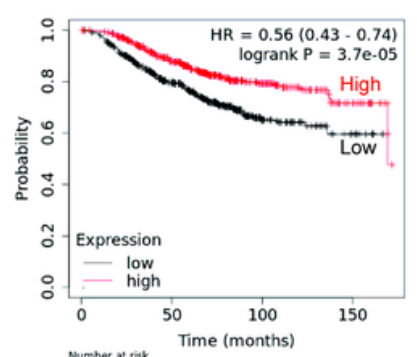

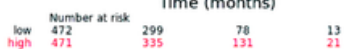

GNG2

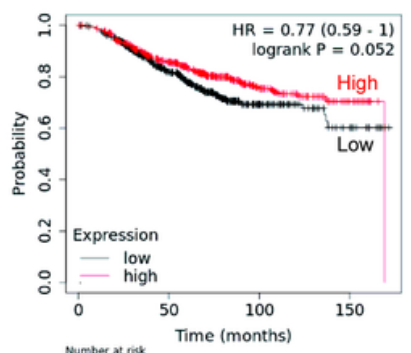

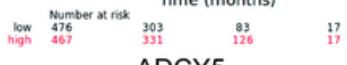

ADCY5

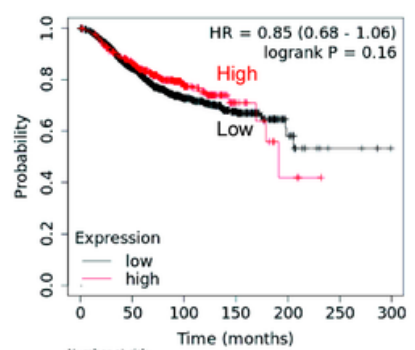

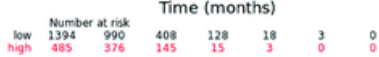

S1PR1

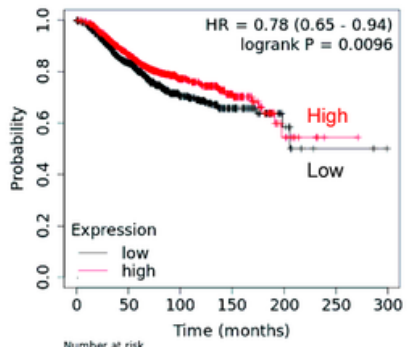

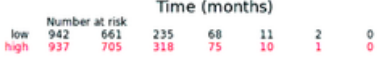

LPAR1

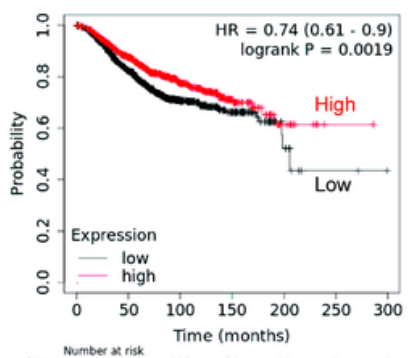

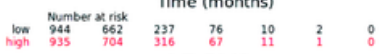

ADCY3

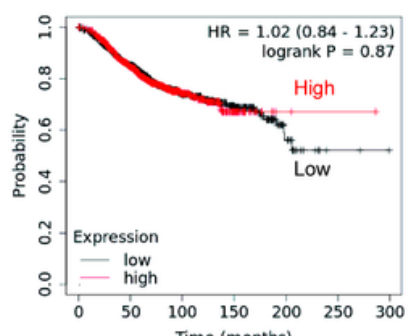

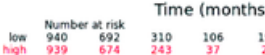

CXCR4

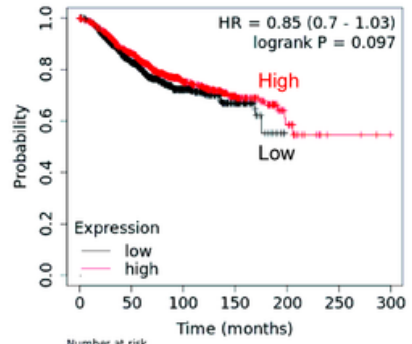

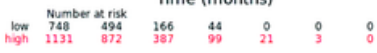

GPR17

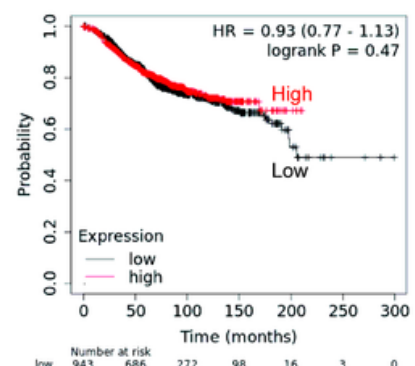

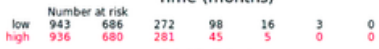

CX3CL1

\section{Figure 7}

A caption was not provided with this version 


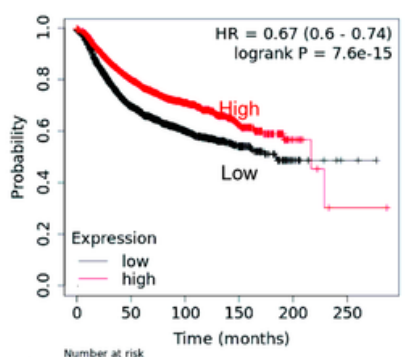

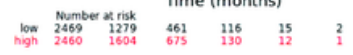

CXCL12

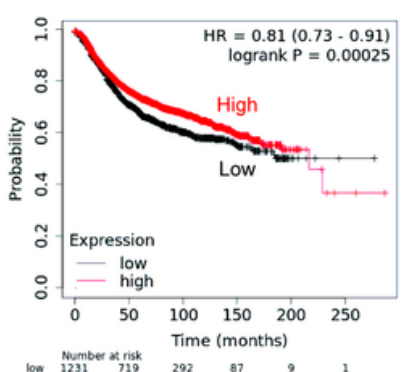

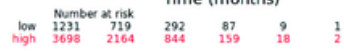

CXCL1

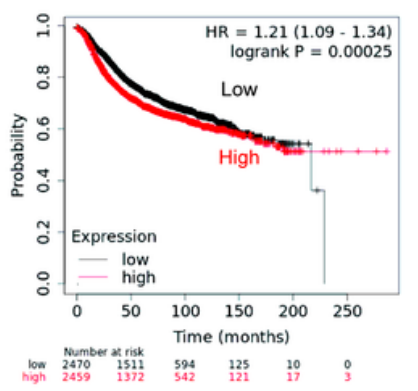

CXCL11

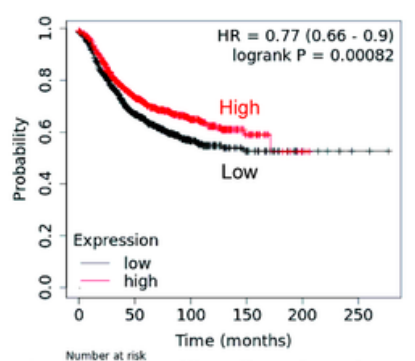

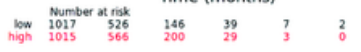

GNG2

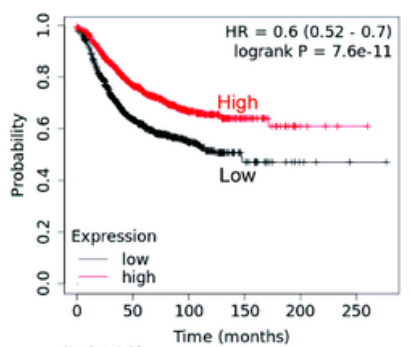

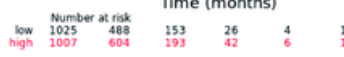

ADCY5

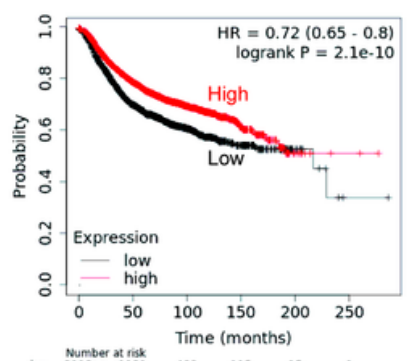

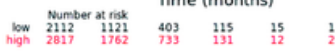

S1PR1

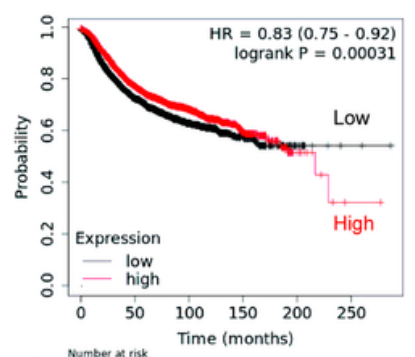

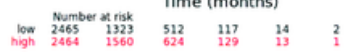

LPAR1

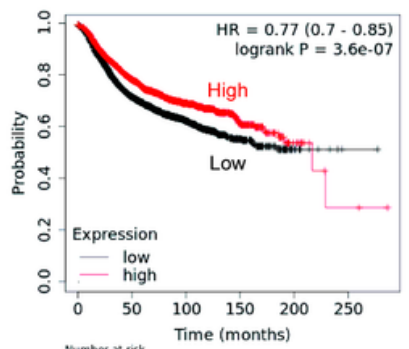

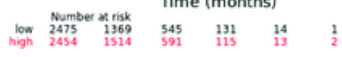

ADCY3

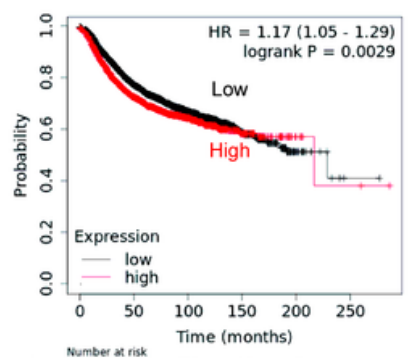

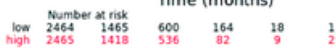

CXCR4

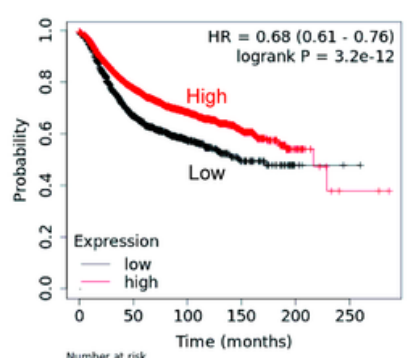

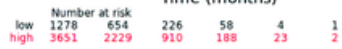

GPR17

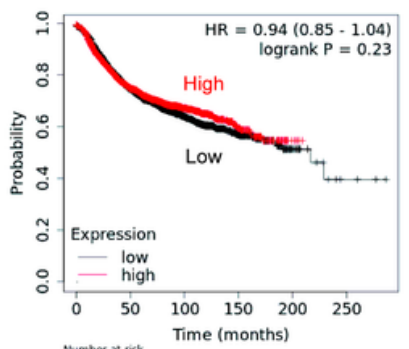

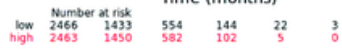

CX3CL1

\section{Figure 8}

A caption was not provided with this version

\section{Supplementary Files}

This is a list of supplementary files associated with this preprint. Click to download.

- SupplementaryTable1.doc

- SupplementaryTable2.doc

- SupplementaryTable3.doc 